\title{
HOMOGENIZATION OF AN ALTERNATING ROBIN-NEUMANN BOUNDARY CONDITION VIA TIME-PERIODIC UNFOLDING
}

\author{
MICOL AMAR, DANIELE ANDREUCCI, AND DARIO BELLAVEGLIA
}

\begin{abstract}
We consider the homogenization of a parabolic problem in a perforated domain with Robin-Neumann boundary conditions oscillating in time. Such oscillations must compensate the blow up of the boundary measure of the holes. We use the technique of time-periodic unfolding in order to obtain a macroscopic parabolic problem containing an extra linear term due to the absorption determined by the Robin condition.
\end{abstract}

Department of Basic and Applied Sciences for Engineering Sapienza University of Rome, Italy

\{micol.amar, daniele.andreucci, dario.bellaveglia\}@sbai.uniroma1.it

Paper dedicated to Professor Nicola Fusco on the occasion of his $60^{\text {th }}$ birthday

\section{INTRODUCTION}

In this paper we study homogenization of a parabolic problem with oscillating coefficients in a domain with holes. That is we look at the problem

$$
a^{\varepsilon}(x, t) \frac{\partial u_{\sigma \tau \varepsilon}}{\partial t}-\operatorname{div}\left(A^{\tau \varepsilon}(x, t) \nabla u_{\sigma \tau \varepsilon}\right)=f,
$$

in the perforated domain, and

$$
A^{\tau \varepsilon}(x, t) \nabla u_{\sigma \tau \varepsilon} \cdot \nu+\alpha_{\sigma \tau}(t) u_{\sigma \tau \varepsilon}=0,
$$

on the boundary of perforations. Here the coefficients have a suitably oscillating character both in time $(\sigma, \tau)$ and in space $(\varepsilon)$. Of course the parabolic problem is completed by boundary and initial data. We are interested in the case where the coefficient $\alpha_{\sigma \tau}$ is a strongly oscillating non-vanishing function in the limit. Our approach is based on the time-periodic unfolding technique introduced in [3]. The operators of time-periodic unfolding are inspired by the operators of space-periodic unfolding introduced and applied in $[11,12,13,14,16,19]$.

Our interest in problems exhibiting oscillations in time originally arised from mathematical models for transport across biological membranes. It has been observed that the pores on the membranes switch between a closed state and an open one, 
either periodically or according to a random scheme (see [1, 9]). A first stochastic model was proposed in [24]. In [6], through homogenization techniques, it was shown that the limiting behavior of problems of this kind sharply depends on the relative scalings of the time and space variables; see also [8] for a MonteCarlo test of the model. In [7] oscillations in the boundary conditions have been coupled to time periodic changes in the diffusivity coefficient as a device to reproduce the selection capability exhibited by biological membranes. A case of sign changing capacity oscillating in space was also treated in $[4,5]$ by means of asymptotic expansions.

In this paper we apply and extend the results of [3] to the case of domains with holes, having in mind a model of cell absorption of a selected protein or drug.

Let us compare our approach here to previous literature. The homogenization of PDEs in perforated domains has been studied widely for many years (see among the others $[17,18,20,22]$ and more recently $[10,12,23]$ and references therein). The homogenization via periodic unfolding of the stationary case in perforated domains with Neumann boundary conditions on the holes has been treated in [12], while the case of Robin boundary conditions was treated in [16]. There the blow up of the area of the holes in the homogenization limit is compensated by the assumption that the coefficient in the boundary condition becomes vanishingly small in the limit. In this paper we deal with an evolutive problem where the coefficient in the boundary data does not tend to zero uniformly as in [16]. Rather, the compensation effect is implied by the switching in time between the Robin condition and a null Neumann condition. This requires a suitable balance between the space and time oscillations.

In Section 2 we introduce the basic definitions and properties of the time-periodic unfolding operator for domains with holes. We identify two possible limiting behaviors depending on the relative magnitude of $\tau$ (the time-period of the oscillations) and $\varepsilon$ (the diameter of the holes and spatial period of the lattice). Notice that this classification relies on the degeneracy of the estimate of the time derivative, whose $L^{p}$-norm we assume to behave in the limit as a parameter $\theta_{\tau}$. We are in the case of fast oscillations (in time) when

$$
\frac{\tau \theta_{\tau}}{\varepsilon} \leq C
$$

and in the case of slow oscillations when

$$
\frac{\tau \theta_{\tau}}{\varepsilon} \rightarrow+\infty
$$

In Section 3 we introduce the oscillating Robin-Neumann parabolic problem and obtain the relevant estimates needed for the homogenization process. There we state precisely our assumptions and introduce a new parameter $\sigma$, that is the fraction of the time-period when the boundary condition is absorbing i.e., of Robin 
type. In Section 4 we deal with the homogenization process in the case

$$
\frac{\sigma}{\varepsilon} \rightarrow \beta \in[0, \infty)
$$

considering the subcases of both fast and slow oscillations. We provide for the homogenized problem, both the two-scale formulation and a more explicit formulation relying upon factorization and cell functions. More precisely we obtain a macroscopic model given by a parabolic equation containing an extra linear term depending on $\alpha_{\sigma \tau}, \beta$ and the geometry. Notice that in our case $\theta_{\tau}=\frac{1}{\sqrt{\tau \varepsilon}}$ so that the case of fast oscillations is defined by $\tau / \varepsilon^{3} \leq C$, while the case of slow oscillations is defined by $\tau / \varepsilon^{3} \rightarrow \infty$. Finally in Section 5 we consider the case

$$
\frac{\sigma}{\varepsilon} \rightarrow \infty
$$

where, essentially, the solution $u_{\tau}$ vanishes in the limit.

\section{The Time-Periodic Unfolding Operator}

2.1. Notation. Throughout the paper $\varepsilon>0$ denotes the space period of the microstructure, and likewise $\tau>0$ and $\sigma>0$ denote its multiscale time periods. Though this is not explicitly stressed by the notation for the sake of simplicity, we always assume that three sequences are given: $\varepsilon_{i} \rightarrow 0, \tau_{i} \rightarrow 0, \sigma_{i} \rightarrow 0$ as $i \rightarrow \infty$. The limiting behavior of quantities depending on $\varepsilon, \tau$ and $\sigma$ is denoted by

$$
\lim _{\tau \rightarrow 0} .
$$

More exactly the parameter $\tau$ represents the time period of time oscillations for example in the boundary condition. The parameter $\varepsilon$ is the spatial period of the microstructure and the characteristic dimension of the perforations of the domain as well. Finally $\sigma$ represents the fraction of the time period $\tau$ in which the Robin absorbing boundary condition is in force. In the following, for the sake of notational simplicity, we index only with $\tau$ some objects that depend on $\varepsilon$ and $\sigma$ too.

2.2. Definitions. Let $\Omega \subset \boldsymbol{R}^{N}$ be a bounded connected open set with Lipschitz boundary, and set

$$
Y=(0,1)^{N}, \quad \Sigma=(0,1), \quad Q=Y \times \Sigma, \quad \Omega_{T}=\Omega \times(0, T) .
$$

Thus $Y$ is the microscopic space cell and $\Sigma$ the microscopic time interval in rescaled coordinates. Next we introduce an open set with Lipschitz boundary $B$ such that $\bar{B} \subset Y$, and define

$$
Y^{*}=Y \backslash \bar{B}, \quad Q^{*}=Y^{*} \times \Sigma .
$$


Considering the tiling of $\boldsymbol{R}^{N}$ given by the sets $\varepsilon(\xi+Y), \xi \in \boldsymbol{Z}^{N}$ we also define

$$
\begin{gathered}
\Xi_{\varepsilon}=\left\{\xi \in Z^{N}, \quad \varepsilon(\xi+Y) \subset \Omega\right\}, \quad \widehat{\Omega}_{\varepsilon}=\text { interior }\left\{\bigcup_{\xi \in \Xi_{\varepsilon}} \varepsilon(\xi+\bar{Y})\right\} ; \\
\widehat{T}_{\tau}=\left\{t \in(0, T) \mid \tau\left(\left[\frac{t}{\tau}\right]+1\right) \leq T\right\}, \quad \Lambda_{\tau}=\widehat{\Omega}_{\varepsilon} \times \widehat{T}_{\tau} .
\end{gathered}
$$

Here and in the definitions below $[r]$ denotes the integer part of $r \in \boldsymbol{R}$. The perforated domain $\Omega^{\varepsilon}$ is defined by

$$
\Omega^{\varepsilon}=\Omega \backslash\left\{\bigcup_{\xi \in \Xi_{\varepsilon}} \varepsilon(\xi+\bar{B})\right\},
$$

and we set $\Omega_{T}^{\varepsilon}=\Omega^{\varepsilon} \times(0, T)$. Moreover we denote

$$
\Gamma^{\varepsilon}=\bigcup_{\xi \in \Xi_{\varepsilon}} \varepsilon(\xi+\partial B), \quad \Gamma_{T}^{\varepsilon}=\Gamma^{\varepsilon} \times(0, T), \quad \boldsymbol{R}_{\varepsilon}^{N}=\boldsymbol{R}^{N} \backslash\left\{\bigcup_{\xi \in \boldsymbol{Z}^{N}} \varepsilon(\xi+B)\right\} .
$$

For $x \in \boldsymbol{R}^{N}$ and $t \in[0,+\infty)$ we define

$$
\left[\frac{x}{\varepsilon}\right]_{Y}=\left(\left[\frac{x_{1}}{\varepsilon}\right], \ldots,\left[\frac{x_{N}}{\varepsilon}\right]\right)
$$

and also denote

$$
x=\varepsilon\left(\left[\frac{x}{\varepsilon}\right]_{Y}+\left\{\frac{x}{\varepsilon}\right\}_{Y}\right), \quad t=\tau\left(\left[\frac{t}{\tau}\right]+\left\{\frac{t}{\tau}\right\}\right) .
$$

Finally for $\sigma$ such that $0<\sigma<1$, define the sets

$$
Q_{\sigma}=Y \times \frac{1}{\sigma} \Sigma, \quad Q_{\sigma}^{*}=Y^{*} \times \frac{1}{\sigma} \Sigma, \quad Q_{\sigma}^{\partial}=\partial B \times \frac{1}{\sigma} \Sigma,
$$

and

$$
A_{\sigma, \tau}=\bigcup_{i=0}^{\left[\frac{T}{\tau}\right]-1}(\tau i, \tau(i+\sigma)) .
$$

For any bounded open set $H$ and any Lebesgue integrable function $w$ in $H$, we denote with $\mathcal{M}_{H}(w)$ the integral average of $w$ with respect to $H$.

The following operator was introduced in [3] (see [11] for the elliptic case)

Definition 2.1 (Time-Periodic Unfolding Operator). For $w$ Lebesgue-measurable in $\Omega_{T}$ the Time-Periodic Unfolding operator $\mathcal{T}_{\tau}$ is defined as

$$
\mathcal{T}_{\tau}(w)(x, t, y, s)= \begin{cases}w\left(\varepsilon\left[\frac{x}{\varepsilon}\right]_{Y}+\varepsilon y, \tau\left[\frac{t}{\tau}\right]+\tau s\right), & (x, t, y, s) \in \Lambda_{\tau} \times Q \\ 0, & \text { otherwise }\end{cases}
$$


In this paper we will mostly work in the set with holes $\Omega^{\varepsilon}$; we define (see [12] for the elliptic case)

Definition 2.2 (Time-Periodic Unfolding Operator in domains with holes). For $w$ Lebesgue-measurable in $\Omega_{T}^{\varepsilon}$ the Time-Periodic Unfolding operator $\mathcal{T}_{\tau}^{*}$ is defined as

$$
\mathcal{T}_{\tau}^{*}(w)(x, t, y, s)= \begin{cases}w\left(\varepsilon\left[\frac{x}{\varepsilon}\right]_{Y}+\varepsilon y, \tau\left[\frac{t}{\tau}\right]+\tau s\right), & (x, t, y, s) \in \Lambda_{\tau} \times Q^{*} \\ 0, & \text { otherwise }\end{cases}
$$

It is an immediate consequence of the definition that if $w_{1}, w_{2}$ are Lebesguemeasurable in $\Omega_{T}^{\varepsilon}$

$$
\mathcal{T}_{\tau}^{*}\left(w_{1} w_{2}\right)=\mathcal{T}_{\tau}^{*}\left(w_{1}\right) \mathcal{T}_{\tau}^{*}\left(w_{2}\right) .
$$

Remark 2.3. Note that for $w$ Lebesgue-measurable in $\Omega_{T}$, the definitions above imply $\mathcal{T}_{\tau}^{*}(w)=\mathcal{T}_{\tau}(w)$ in $\Lambda_{\tau} \times Q^{*}$.

Definition 2.4 (Two Time Scales-Periodic Unfolding Operator). For $w$ Lebesguemeasurable in $\Omega_{T}^{\varepsilon}$ we define the operator $\mathcal{T}_{\tau, \sigma}^{*}$ as $\mathcal{T}_{\tau, \sigma}^{*}(w)(x, t, y, r)=\mathcal{T}_{\tau}^{*}(w)(x, t, y, \sigma r)$, for $(x, t, y, r) \in \Lambda_{\tau} \times Q_{\sigma}^{*}$.

Definition 2.5 (Boundary Two Time Scales-Periodic Unfolding Operator). For $w$ Lebesgue-measurable on $\Gamma_{T}^{\varepsilon}$ we define the operator $\mathcal{T}_{\tau, \sigma}^{b}$ as

$$
\mathcal{T}_{\tau, \sigma}^{b}(w)(x, t, y, r)= \begin{cases}w\left(\varepsilon\left[\frac{x}{\varepsilon}\right]_{Y}+\varepsilon y, \tau\left[\frac{t}{\tau}\right]+\tau \sigma r\right), & (x, t, y, r) \in \Lambda_{\tau} \times Q_{\sigma}^{\partial} \\ 0, & \text { otherwise }\end{cases}
$$

Note that $\mathcal{T}_{\tau, \sigma}^{b}(w)$ is the trace of $\mathcal{T}_{\tau, \sigma}^{*}(w)$ on $\partial B$ if both are defined.

Remark 2.6. Note that analogues of property (2.1) are also satisfied by the operators $\mathcal{T}_{\tau, \sigma}^{*}$ and $\mathcal{T}_{\tau, \sigma}^{b}$.

2.3. Basic Properties of the Operators. In this Subsection we collect some properties of the operators defined in Subsection 2.2. We refer the reader to [3] for many proofs which stay essentially unchanged in this case, and which rely essentially on $[11,12]$.

In the following $p \in[1, \infty)$ unless otherwise noted. Also functions depending on the microscopic variables $(y, s, r)$, or only on $(x, t)$ are often considered trivially extended to the appropriate macro-microscopic domain. 
Proposition 2.7. The operator $\mathcal{T}_{\tau}^{*}: L^{p}\left(\Omega_{T}^{\varepsilon}\right) \rightarrow L^{p}\left(\Omega_{T} \times Q^{*}\right)$ is linear and continuous.

In addition for all $w \in L^{p}\left(\Omega_{T}^{\varepsilon}\right)$ we have

$$
\left\|\mathcal{T}_{\tau}^{*}(w)\right\|_{L^{p}\left(\Omega_{T} \times Q^{*}\right)} \leq\|w\|_{L^{p}\left(\Omega_{T}^{\varepsilon}\right)},
$$

and

$$
\left|\int_{\Omega_{T}^{\varepsilon}} w \mathrm{~d} x \mathrm{~d} t-\iint_{\Omega_{T} \times Q^{*}} \mathcal{T}_{\tau}^{*}(w) \mathrm{d} x \mathrm{~d} t \mathrm{~d} y \mathrm{~d} s\right| \leq \int_{\Omega_{T}^{\varepsilon} \backslash \Lambda_{\tau}}|w| \mathrm{d} x \mathrm{~d} t .
$$

Lemma 2.8. Let $\phi \in W^{1,1}\left(\Omega_{T}^{\varepsilon} \times Q^{*}\right)$, and define

$$
\phi^{\tau}(x, t)=\phi\left(x, t, \frac{x}{\varepsilon}, \frac{t}{\tau}\right), \quad(x, t) \in \Omega_{T}^{\varepsilon},
$$

where $\phi$ has been extended by $Q$-periodicity to $\Omega_{T}^{\varepsilon} \times \boldsymbol{R}_{\varepsilon}^{N} \times \boldsymbol{R}$. Then in $\Omega_{T} \times Q^{*}$

$$
\frac{\partial}{\partial s} \mathcal{T}_{\tau}^{*}\left(\phi^{\tau}\right)=\tau \mathcal{T}_{\tau}^{*}\left(\frac{\partial \phi}{\partial t}\right)+\mathcal{T}_{\tau}^{*}\left(\frac{\partial \phi}{\partial s}\right)
$$

and

$$
\nabla_{y} \mathcal{T}_{\tau}^{*}\left(\phi^{\tau}\right)=\varepsilon \mathcal{T}_{\tau}^{*}\left(\nabla_{x} \phi\right)+\mathcal{T}_{\tau}^{*}\left(\nabla_{y} \phi\right)
$$

If we instead define $\phi^{\sigma \tau} \in W^{1,1}\left(\Omega_{T}^{\varepsilon} \times Q_{\sigma}^{*}\right)$ as

$$
\phi^{\sigma \tau}(x, t)=\phi\left(x, t, \frac{x}{\varepsilon}, \frac{t}{\sigma \tau}\right), \quad(x, t) \in \Omega_{T}^{\varepsilon},
$$

where $\phi$ has been extended by $Q_{\sigma}$-periodicity to $\Omega_{T}^{\varepsilon} \times \boldsymbol{R}_{\varepsilon}^{N} \times \boldsymbol{R}$, then in $\Omega_{T} \times Q_{\sigma}^{*}$

$$
\frac{\partial}{\partial r} \mathcal{T}_{\tau, \sigma}^{*}\left(\phi^{\sigma \tau}\right)=\sigma \tau \mathcal{T}_{\tau, \sigma}^{*}\left(\frac{\partial \phi}{\partial t}\right)+\mathcal{T}_{\tau, \sigma}^{*}\left(\frac{\partial \phi}{\partial r}\right)
$$

Also $\mathcal{T}_{\tau, \sigma}^{*}$ (respectively $\mathcal{T}_{\tau, \sigma}^{b}$ ) satisfies an analogue of (2.6) (respectively (2.8)) in $\Omega_{T} \times Q_{\sigma}^{*}$ (respectively in $\Omega_{T} \times Q_{\sigma}^{\partial}$ ).

Proposition 2.9. For $\phi$ measurable in $Q^{*}$, extended by $Q$-periodicity, define the sequence

$$
\phi^{\tau}(x, t)=\phi\left(\frac{x}{\varepsilon}, \frac{t}{\tau}\right), \quad(x, t) \in \boldsymbol{R}_{\varepsilon}^{N} \times \boldsymbol{R} .
$$

Then

$$
\mathcal{T}_{\tau}^{*}\left(\phi^{\tau}\right)(x, t, y, s)= \begin{cases}\phi(y, s), & (x, t, y, s) \in \Lambda_{\tau} \times Q^{*} \\ 0, & \text { otherwise. }\end{cases}
$$

Moreover, if $\phi \in L^{p}\left(Q^{*}\right)$ as $\tau \rightarrow 0$

$$
\mathcal{T}_{\tau}^{*}\left(\phi^{\tau}\right) \rightarrow \phi, \quad \text { strongly in } L^{p}\left(\Omega_{T} \times Q^{*}\right) \text {. }
$$


If $\phi$ has $\nabla_{y} \phi$ and $\frac{\partial \phi}{\partial s}$ in $L^{p}\left(Q^{*}\right)$ then

$$
\begin{aligned}
& \nabla_{y}\left(\mathcal{T}_{\tau}^{*}\left(\phi^{\tau}\right)\right) \rightarrow \nabla_{y} \phi, \quad \text { strongly in } L^{p}\left(\Omega_{T} \times Q^{*}\right) . \\
& \frac{\partial}{\partial s}\left(\mathcal{T}_{\tau}^{*}\left(\phi^{\tau}\right)\right) \rightarrow \frac{\partial}{\partial s} \phi, \quad \text { strongly in } L^{p}\left(\Omega_{T} \times Q^{*}\right) .
\end{aligned}
$$

Proposition 2.10. For $\phi$ measurable on $Q_{\sigma}^{\partial}$, extended by $Y$ and $\frac{1}{\sigma} \Sigma$-periodicity, define the sequence

$$
\phi^{\sigma \tau}(x, t)=\phi\left(\frac{x}{\varepsilon}, \frac{1}{\sigma}\left\{\frac{t}{\tau}\right\}\right), \quad(x, t) \in \partial \boldsymbol{R}_{\varepsilon}^{N} \times \boldsymbol{R} .
$$

Assume also $\operatorname{supp} \phi \subset \partial B \times \Sigma$. Then

$$
\mathcal{T}_{\tau, \sigma}^{b}\left(\phi^{\sigma \tau}\right)(x, t, y, r)= \begin{cases}\phi(y, r), & (x, t, y, r) \in \Lambda_{\tau} \times \partial B \times \Sigma, \\ 0, & \text { otherwise. }\end{cases}
$$

Moreover, if $\phi \in L^{p}(\partial B \times \Sigma)$ as $\tau \rightarrow 0$

$$
\mathcal{T}_{\tau, \sigma}^{b}\left(\phi^{\sigma \tau}\right) \rightarrow \phi, \quad \text { strongly in } L^{p}\left(\Omega_{T} \times \partial B \times \Sigma\right) .
$$

The proof of Proposition 2.10 follows straightforwardly from the definitions. Notice that $\phi^{\sigma \tau}$ is periodic in time with period $\tau$.

Next we recall the following results on the operator $\mathcal{T}_{\tau}$ and converging sequences.

Proposition 2.11. Let $\left\{w_{\tau}\right\}$ be a sequence of functions in $L^{p}\left(\Omega_{T}\right)$.

If $w_{\tau} \rightarrow w$ strongly in $L^{p}\left(\Omega_{T}\right)$ as $\tau \rightarrow 0$, then

$$
\mathcal{T}_{\tau}\left(w_{\tau}\right) \rightarrow w(x, t), \quad \text { strongly in } L^{p}\left(\Omega_{T} \times Q\right) .
$$

Proposition 2.12. If $w \in L^{p}\left(\Omega_{T}\right)$, then as $\tau \rightarrow 0$

$$
\mathcal{T}_{\tau}^{*}(w) \rightarrow w, \quad \text { strongly in } L^{p}\left(\Omega_{T} \times Q^{*}\right) .
$$

If $w_{\tau}$ is a bounded sequence of functions in $L^{p}\left(\Omega_{T}^{\varepsilon}\right), p>1$, then up to subsequences

$$
\mathcal{T}_{\tau}^{*}\left(w_{\tau}\right) \rightarrow \widehat{w}, \quad \text { weakly in } L^{p}\left(\Omega_{T} \times Q^{*}\right),
$$

for a suitable function $\widehat{w} \in L^{p}\left(\Omega_{T} \times Q^{*}\right)$.

Proposition 2.13. If $w \in \operatorname{Lip}\left(\Omega_{T}\right)$, then

$$
\left\|\mathcal{T}_{\tau, \sigma}^{b}(w)-w\right\|_{L^{\infty}\left(\Lambda_{\tau} \times Q_{\sigma}^{\partial}\right)} \leq \sqrt{N} \varepsilon\|\nabla w\|_{L^{\infty}\left(\Omega_{T}\right)}+\tau\left\|w_{t}\right\|_{L^{\infty}\left(\Omega_{T}\right)},
$$

which implies

$$
\mathcal{T}_{\tau, \sigma}^{b}(w) \rightarrow w, \quad \text { strongly in } L^{p}\left(\Omega_{T} \times K\right),
$$

for any bounded set $K \subset \partial B \times \boldsymbol{R}$.

The proof of Proposition 2.13 follows from standard arguments (see also [14] (4.15)). 
Proposition 2.14. Let $\left\{w_{\tau}\right\}$ be a sequence bounded in $L^{p}\left(0, T, W^{1, p}\left(\Omega^{\varepsilon}\right)\right)$, and also satisfying the estimate

$$
\left\|\frac{\partial w_{\tau}}{\partial t}\right\|_{L^{p}\left(\Omega_{T}^{\varepsilon}\right)} \leq \theta_{\tau}, \quad \text { with } \tau \theta_{\tau} \leq \gamma .
$$

Then, up to a subsequence, there exists a function $w \in L^{p}\left(\Omega_{T} \times \Sigma\right)$ such that

$$
\mathcal{T}_{\tau}^{*}\left(w_{\tau}\right) \rightarrow w, \quad \text { weakly in } L^{p}\left(\Omega_{T}, W^{1, p}\left(Q^{*}\right)\right) .
$$

Moreover, if

$$
\lim _{\tau \rightarrow 0} \tau \theta_{\tau}=0,
$$

then $w=w(x, t) \in L^{p}\left(\Omega_{T}\right)$.

Proof. The proof is analogue to the one of Proposition 2.12 in [3]. In practice in [3] we considered the special case where the quantity $\theta_{\tau}$ appearing in $(2.19)$ equals $\tau^{-m}$ with $0 \leq m<1$.

Remark 2.15. In our application, the quantity $\theta_{\tau}$ becomes unbounded in the limit $\tau \rightarrow 0$.

As for the operator $\mathcal{T}_{\tau}^{*}$, we have

Proposition 2.16. The operator $\mathcal{T}_{\tau, \sigma}^{*}: L^{p}\left(\Omega_{T}^{\varepsilon}\right) \rightarrow L^{p}\left(\Omega_{T} \times Q_{\sigma}^{*}\right)$ is linear and continuous.

In addition for all $w \in L^{p}\left(\Omega_{T}^{\varepsilon}\right)$ we have

$$
\left\|\mathcal{T}_{\tau, \sigma}^{*}(w)\right\|_{L^{p}\left(\Omega_{T} \times Q_{\sigma}^{*}\right)} \leq \frac{1}{\sigma^{\frac{1}{p}}}\|w\|_{L^{p}\left(\Omega_{T}^{\varepsilon}\right)}
$$

and

$$
\left|\int_{\Omega_{T}^{\varepsilon}} w \mathrm{~d} x \mathrm{~d} t-\sigma \int_{\Omega_{T} \times Q_{\sigma}^{*}} \mathcal{T}_{\tau, \sigma}^{*}(w) \mathrm{d} x \mathrm{~d} t \mathrm{~d} y \mathrm{~d} r\right| \leq \int_{\Omega_{T}^{\varepsilon} \backslash \Lambda_{\tau}}|w| \mathrm{d} x \mathrm{~d} t .
$$

Proposition 2.17. The operator $\mathcal{T}_{\tau, \sigma}^{b}: L^{p}\left(\Gamma_{T}^{\varepsilon}\right) \rightarrow L^{p}\left(\Omega_{T} \times Q_{\sigma}^{\partial}\right)$ is linear and continuous.

In addition for all $w \in L^{p}\left(\Gamma_{T}^{\varepsilon}\right)$ we have

$$
\left\|\mathcal{T}_{\tau, \sigma}^{b}(w)\right\|_{L^{p}\left(\Omega_{T} \times Q_{\sigma}^{\partial}\right)} \leq\left(\frac{\varepsilon}{\sigma}\right)^{\frac{1}{p}}\|w\|_{L^{p}\left(\Gamma_{T}^{\varepsilon}\right)}
$$

and

$$
\left|\int_{\Gamma_{T}^{\varepsilon}} w \mathrm{~d} \sigma_{x} \mathrm{~d} t-\frac{\sigma}{\varepsilon} \int_{\Omega_{T} \times Q_{\sigma}^{\partial}} \mathcal{T}_{\tau, \sigma}^{b}(w) \mathrm{d} x \mathrm{~d} t \mathrm{~d} \sigma_{y} \mathrm{~d} r\right| \leq \int_{\Gamma_{T}^{\varepsilon} \backslash \Lambda_{\tau}}|w| \mathrm{d} \sigma_{x} \mathrm{~d} t .
$$


Remark 2.18. From Propositions 2.7, 2.16 and 2.17 and Lemma 2.8, for any suitably regular $w: \Omega_{T}^{\varepsilon} \rightarrow \boldsymbol{R}$ it follows

$$
\begin{aligned}
\left\|\nabla_{y} \mathcal{T}_{\tau}^{*}\left(w_{\tau}\right)\right\|_{L^{p}\left(\Omega_{T} \times Q^{*}\right)} & \leq \varepsilon\left\|\nabla_{x} w_{\tau}\right\|_{L^{p}\left(\Omega_{T}^{\varepsilon}\right)}, \\
\left\|\frac{\partial}{\partial s} \mathcal{T}_{\tau}^{*}\left(w_{\tau}\right)\right\|_{L^{p}\left(\Omega_{T} \times Q^{*}\right)} & \leq \tau\left\|\frac{\partial w_{\tau}}{\partial t}\right\|_{L^{p}\left(\Omega_{T}^{\varepsilon}\right)}, \\
\left\|\nabla_{y} \mathcal{T}_{\tau, \sigma}^{*}\left(w_{\tau}\right)\right\|_{L^{p}\left(\Omega_{T} \times Q_{\sigma}^{*}\right)} & \leq \frac{\varepsilon}{\sigma^{\frac{1}{p}}}\left\|\nabla_{x} w_{\tau}\right\|_{L^{p}\left(\Omega_{T}^{\varepsilon}\right)}, \\
\left\|\frac{\partial}{\partial r} \mathcal{T}_{\tau, \sigma}^{*}\left(w_{\tau}\right)\right\|_{L^{p}\left(\Omega_{T} \times Q_{\sigma}^{*}\right)} & \leq \tau \sigma^{1-\frac{1}{p}}\left\|\frac{\partial w_{\tau}}{\partial t}\right\|_{L^{p}\left(\Omega_{T}^{\varepsilon}\right)}, \\
\left\|\frac{\partial}{\partial r} \mathcal{T}_{\tau, \sigma}^{b}\left(w_{\tau}\right)\right\|_{L^{p}\left(\Omega_{T} \times Q_{\sigma}^{\partial}\right)} & \leq \varepsilon^{\frac{1}{p}} \tau \sigma^{1-\frac{1}{p}}\left\|\frac{\partial w_{\tau}}{\partial t}\right\|_{L^{p}\left(\Gamma_{T}^{\varepsilon}\right)}
\end{aligned}
$$

The following results essentially state that, under assumptions (2.19), (2.21) the dilation by $1 / \sigma$ transforming $\mathcal{T}_{\tau}^{*}$ to $\mathcal{T}_{\tau, \sigma}^{*}$ does not destroy the weak convergence in (2.20). Notice that this applies in the domain $Q^{*}$ not changing with $\sigma$.

Lemma 2.19. Let $\left\{w_{\tau}\right\}$ be a sequence bounded in $L^{p}\left(0, T ; W^{1, p}\left(\Omega^{\varepsilon}\right)\right), p>1$ and also satisfying the estimate (2.19), and (2.21). Then, up to a subsequence

$$
\mathcal{T}_{\tau, \sigma}^{*}\left(w_{\tau}\right) \rightarrow w, \quad \text { weakly in } L^{p}\left(\Omega_{T} \times Q^{*}\right),
$$

where $w=w(x, t)$ is the same weak limit as in (2.20).

Proof. From Proposition 2.14 we know that $\mathcal{T}_{\tau}^{*}\left(w_{\tau}\right)$ converges weakly to $w(x, t)$ in $L^{p}\left(\Omega_{T} ; W^{1, p}\left(Q^{*}\right)\right)$. Fix $\varphi \in L^{\frac{p}{p-1}}\left(\Omega_{T} \times Q^{*}\right)$ and define

$$
J_{\tau}(s)=\int_{\Omega_{T}} \int_{Y^{*}}\left[\mathcal{T}_{\tau}^{*}\left(w_{\tau}\right)(x, t, y, s)-w(x, t)\right]\left(\int_{\Sigma} \varphi(x, t, y, r) \mathrm{d} r\right) \mathrm{d} x \mathrm{~d} t \mathrm{~d} y, \quad s \in \Sigma .
$$

Owing to (2.27) we know that $J_{\tau}$ is continuous in $\Sigma$ for each $\tau>0$. Fix arbitrarily $\delta>0$. The asserted weak convergence guarantees that a $\tau_{\delta}>0$ exists such that

$$
\left|\int_{\Sigma} J_{\tau}(s) \mathrm{d} s\right| \leq \delta, \quad 0<\tau<\tau_{\delta} .
$$

It is easily seen that the continuity of $J_{\tau}$ then implies the existence of a point $s^{*} \in \Sigma$, possibly depending on $\tau$, such that

$$
\left|J_{\tau}\left(s^{*}\right)\right| \leq|\Sigma|^{-1} \delta, \quad 0<\tau<\tau_{\delta} .
$$

Next we calculate

$$
\int_{\Omega_{T}} \int_{Q^{*}}\left[\mathcal{T}_{\tau, \sigma}^{*}\left(w_{\tau}\right)(x, t, y, r)-w(x, t)\right] \varphi(x, t, y, r) \mathrm{d} x \mathrm{~d} t \mathrm{~d} y \mathrm{~d} r=J^{1}+J_{\tau}\left(s^{*}\right),
$$


where

$$
\begin{aligned}
J^{1}= & \int_{\Omega_{T}} \int_{Q^{*}}\left[\mathcal{T}_{\tau, \sigma}^{*}\left(w_{\tau}\right)(x, t, y, r)-\mathcal{T}_{\tau}^{*}\left(w_{\tau}\right)\left(x, t, y, s^{*}\right)\right] \varphi(x, t, y, r) \mathrm{d} x \mathrm{~d} t \mathrm{~d} y \mathrm{~d} r= \\
& \int_{\Omega_{T}} \int_{Y^{*}} \frac{1}{\sigma} \int_{0}^{\sigma}\left[\mathcal{T}_{\tau}^{*}\left(w_{\tau}\right)(x, t, y, s)-\mathcal{T}_{\tau}^{*}\left(w_{\tau}\right)\left(x, t, y, s^{*}\right)\right] \varphi\left(x, t, y, \frac{s}{\sigma}\right) \mathrm{d} x \mathrm{~d} t \mathrm{~d} y \mathrm{~d} s .
\end{aligned}
$$

Clearly the statement will follow if we are able to prove that $J^{1} \rightarrow 0$ as $\tau \rightarrow 0$. Indeed we estimate

$$
\begin{aligned}
\left|J^{1}\right| & \leq \frac{1}{\sigma}\left(\int_{\Omega_{T}} \int_{Y^{*}} \int_{0}^{\sigma}\left|\mathcal{T}_{\tau}^{*}\left(w_{\tau}\right)(x, t, y, s)-\mathcal{T}_{\tau}^{*}\left(w_{\tau}\right)\left(x, t, y, s^{*}\right)\right|^{p} \mathrm{~d} x \mathrm{~d} t \mathrm{~d} y \mathrm{~d} s\right)^{\frac{1}{p}} \times \\
& \left(\sigma \iint_{\Omega_{T}^{*}}|\varphi(x, t, y, r)|^{\frac{p}{p-1}} \mathrm{~d} x \mathrm{~d} t \mathrm{~d} y \mathrm{~d} r\right)^{\frac{p-1}{p}} \\
& \leq \gamma \frac{1}{\sigma^{\frac{1}{p}}}\left(\int_{\Omega_{T}} \int_{Y^{*}} \int_{0}^{\sigma}\left|\int_{s^{*}}^{s} \frac{\partial}{\partial z} \mathcal{T}_{\tau}^{*}\left(w_{\tau}\right)(x, t, y, z) \mathrm{d} z\right|^{p} \mathrm{~d} x \mathrm{~d} t \mathrm{~d} y \mathrm{~d} s\right)^{\frac{1}{p}} \\
& \leq \gamma \frac{1}{\sigma^{\frac{1}{p}}}\left(\int_{\Omega_{T}} \int_{Y^{*}} \int_{0}^{\sigma}\left(\int_{s^{*}}^{s}\left|\frac{\partial}{\partial z} \mathcal{T}_{\tau}^{*}\left(w_{\tau}\right)(x, t, y, z)\right|^{p} \mathrm{~d} z\right)^{\mathrm{d}} x \mathrm{~d} t \mathrm{~d} y \mathrm{~d} s\right)^{\frac{1}{p}} \\
& \leq \gamma\left(\left.\int_{\Omega_{T}} \int_{Q^{*}} \frac{\partial}{\partial z} \mathcal{T}_{\tau}^{*}\left(w_{\tau}\right)(x, t, y, z)\right|^{p} \mathrm{~d} x \mathrm{~d} t \mathrm{~d} y \mathrm{~d} z\right)^{\frac{1}{p}} \quad \\
& \leq \gamma \tau\left(\int_{\Omega_{T}} \int_{Q^{*}}\left|\mathcal{T}_{\tau}^{*}\left(\frac{\partial}{\partial t} w_{\tau}\right)(x, t, y, z)\right|^{p} \mathrm{~d} x \mathrm{~d} t \mathrm{~d} y \mathrm{~d} z\right)^{\frac{1}{p}} \leq \gamma \tau \theta_{\tau} \rightarrow 0
\end{aligned}
$$

where we have made use again of (2.5), (2.19) and (2.21).

Then we consider the traces.

Proposition 2.20. Let $\left\{w_{\tau}\right\}$ be a sequence bounded in $L^{p}\left(0, T, W^{1, p}\left(\Omega^{\varepsilon}\right)\right)$ with $p>1$, and also satisfying the estimate (2.19) and (2.21). Assume also

$$
\left\|w_{\tau}\right\|_{L^{\infty}\left(\Omega_{T}^{\varepsilon}\right)} \leq C,
$$

and

$$
\frac{\varepsilon}{\sigma^{\frac{1}{p}}} \leq C
$$


where $0<C<\infty$ is a constant independent of $\tau, \varepsilon$ and $\sigma$. Then up to a subsequence

$$
\mathcal{T}_{\tau, \sigma}^{b}\left(w_{\tau}\right) \rightarrow w, \quad \text { weakly in } L^{p}\left(\Omega_{T} \times \partial B \times \Sigma\right),
$$

where $w=w(x, t)$ is the same weak limit as in (2.31) and (2.20).

Proof. The hypothesis (2.34) implies $\left|\mathcal{T}_{\tau, \sigma}^{b}\left(w_{\tau}\right)\right| \leq C$ and then, up to a subsequence

$$
\mathcal{T}_{\tau, \sigma}^{b}\left(w_{\tau}\right) \rightarrow W, \quad \text { weakly in } L^{p}\left(\Omega_{T} \times \partial B \times \Sigma\right) .
$$

Then applying Lemma 2.19, and inequality (2.28), in view of (2.35), the trace theorem applied to $\mathcal{T}_{\tau, \sigma}^{*}\left(w_{\tau}\right)$ yields, for any $\varphi \in C^{1}\left(\overline{\Omega_{T} \times Q}\right)$ such that $\operatorname{supp} \varphi \subset$ $\Omega_{T} \times Q$

$$
\begin{gathered}
\int_{\Omega_{T}} \int_{\partial B} \int_{\Sigma} W \varphi \nu_{i} \mathrm{~d} x \mathrm{~d} t \mathrm{~d} \sigma_{y} \mathrm{~d} r= \\
\lim _{\tau \rightarrow 0} \int_{\Omega_{T}} \int_{\partial B} \int_{\Sigma} \mathcal{T}_{\tau, \sigma}^{b}\left(w_{\tau}\right) \varphi \nu_{i} \mathrm{~d} x \mathrm{~d} t \mathrm{~d} \sigma_{y} \mathrm{~d} r= \\
\lim _{\tau \rightarrow 0} \iint_{\Omega_{T}} \frac{\partial}{\partial y_{i}}\left(\mathcal{T}_{\tau, \sigma}^{*}\left(w_{\tau}\right) \varphi\right) \mathrm{d} x \mathrm{~d} t \mathrm{~d} y \mathrm{~d} r= \\
\int_{\Omega_{T}} \int_{Q^{*}} \frac{\partial}{\partial y_{i}}(w \varphi) \mathrm{d} x \mathrm{~d} t \mathrm{~d} y \mathrm{~d} r=\int_{\Omega_{T}} \int_{\partial B} \int_{\Sigma} w \varphi \nu_{i} \mathrm{~d} x \mathrm{~d} t \mathrm{~d} \sigma_{y} \mathrm{~d} r
\end{gathered}
$$

for $i=1, \cdots, N$, where $\nu=\left(\nu_{i}\right)$ is the interior normal to $\partial B$. Then (2.37) implies $W(x, t, y, r)=w(x, t)$ in $\Omega_{T} \times \partial B \times \Sigma$.

Under general assumptions on the parameters we are able to prove the following Theorem, to be compared with Theorem 2.24.

Theorem 2.21. Let $p>1$ and $\left\{w_{\tau}\right\}$ be a sequence bounded in in $L^{p}\left(0, T, W^{1, p}\left(\Omega^{\varepsilon}\right)\right)$. If $\mathcal{T}_{\tau}^{*}\left(w_{\tau}\right) \rightarrow w$ weakly in $L^{p}\left(\Omega_{T}, W^{1, p}\left(Q^{*}\right)\right)$ with $w \in L^{p}\left(0, T ; W^{1, p}(\Omega)\right)$ then up to a subsequence, there exists $\left.\widetilde{w} \in L^{p}\left(\Omega_{T} \times \Sigma ; W_{\text {per }}^{1, p}\left(Y^{*}\right)\right)\right)$ such that $\mathcal{M}_{Y^{*}}(\widetilde{w})=0$, and as $\tau \rightarrow 0$

$$
\mathcal{T}_{\tau}^{*}\left(\nabla w_{\tau}\right) \rightarrow \nabla w+\nabla_{y} \widetilde{w} \quad \text { weakly in } L^{p}\left(\Omega_{T} \times Q^{*}\right) .
$$

Proof. See Theorem 2.11 in [3] and, for the elliptic case, Theorem 3.5 in [11].

Remark 2.22. Even if Theorem 2.21 holds true for all the possible combinations of parameters $\tau, \varepsilon$ and $\sigma$, we use this result only in the case of slow oscillations defined by

$$
\lim _{\tau \rightarrow 0} \frac{\tau \theta_{\tau}}{\varepsilon}=+\infty
$$

for $\theta_{\tau}$ as in (2.19). In the other cases (fast oscillations) we use the results in Subsection 2.4. 
Remark 2.23. In our application $p=2, \theta_{\tau}=1 / \sqrt{\tau \varepsilon}$ and we need in general Proposition 2.20 and specifically (2.21) and (2.35) to hold true. To achieve this, taking into account (2.39), we have to restrict ourselves to the range $\tau \ll \varepsilon$ and $\varepsilon \leq \gamma \sqrt{\sigma}$. In the case of slow oscillations in practice we further assume $\tau \gg \varepsilon^{3}$ (see the assumptions of Theorem 4.3).

2.4. Fast Oscillations. The case of fast oscillations is defined by the condition

$$
\frac{\tau}{\varepsilon} \theta_{\tau} \leq C
$$

where $C$ is a constant independent of $\tau, \varepsilon$ and $\sigma$. In this case we prove the following

Theorem 2.24. Let $p>1$ and $\left\{w_{\tau}\right\}$ be a sequence bounded in $L^{p}\left(0, T ; W^{1, p}\left(\Omega^{\varepsilon}\right)\right)$ and satisfying the estimate $(2.19)$. If $\mathcal{T}_{\tau}^{*}\left(w_{\tau}\right) \rightarrow w$ weakly in $L^{p}\left(\Omega_{T} ; W^{1, p}\left(Q^{*}\right)\right)$ with $w \in L^{p}\left(0, T ; W^{1, p}(\Omega)\right)$ and $(2.40)$ holds true with

$$
\lim _{\tau \rightarrow 0} \theta_{\tau} \rightarrow \infty,
$$

then up to a subsequence there exists $\widehat{w} \in L^{p}\left(\Omega_{T} ; W_{\text {per }}^{1, p}\left(Q^{*}\right)\right)$ such that $\mathcal{M}_{Q^{*}}(\widehat{w})=0$ and, as $\tau \rightarrow 0$

$$
\begin{aligned}
\mathcal{T}_{\tau}^{*}\left(\nabla w_{\tau}\right) & \rightarrow \nabla w+\nabla_{y} \widehat{w} & \text { weakly in } L^{p}\left(\Omega_{T} \times Q^{*}\right), \\
\frac{\tau}{\varepsilon} \mathcal{T}_{\tau}^{*}\left(\frac{\partial}{\partial t} w_{\tau}\right) & \rightarrow \frac{\partial \widehat{w}}{\partial s} & \text { weakly in } L^{p}\left(\Omega_{T} \times Q^{*}\right) .
\end{aligned}
$$

If we replace hypotheses (2.40), (2.41) with

$$
\lim _{\tau \rightarrow 0} \frac{\tau}{\varepsilon} \theta_{\tau}=0,
$$

allowing $\theta_{\tau}$ to stay bounded as $\tau \rightarrow 0$, then the limit relations (2.42) and (2.43) still hold true and

$$
\frac{\partial \widehat{w}}{\partial s}=0
$$

so that $\widehat{w}=\widehat{w}(x, y, t)$ does not depend on $s$.

Proof. See Theorems 2.16 and 2.18 in [3].

\section{The Oscillating Robin-Neumann Parabolic Problem}

3.1. Statement of the problem and assumptions. The requirements of this subsection will be assumed in Sections 4 and 5 without further reference.

We need that

$$
\lim _{\tau \rightarrow 0} \frac{\tau}{\varepsilon}=0 .
$$

Let $a: \Omega_{T} \times Y \rightarrow \boldsymbol{R}$ be a measurable function. We assume that $a$ satisfy the uniform estimates

$$
0<C^{-1} \leq a \leq C<\infty
$$


for some $C>1$. Let then

$$
A^{\tau}(x, t)=\mathcal{A}^{\tau}\left(x, t, \frac{x}{\varepsilon}, \frac{t}{\tau}\right), \quad \mathcal{A}^{\tau}=\mathcal{A}^{\tau}(x, t, y, s),
$$

be a sequence of $N \times N$ matrices such that for all $\tau>0$

$$
\left\|\mathcal{A}_{i j}^{\tau}\right\|_{\infty} \leq C, \quad i, j=1, \ldots, N ; \quad \mathcal{A}^{\tau} \xi \cdot \xi \geq C^{-1}|\xi|^{2}, \quad \xi \in \boldsymbol{R}^{N} .
$$

We also assume that $a$ is Lipschitz continuous in $t$ and that

$$
\left|\frac{\partial a}{\partial t}\right|, \quad\left|\frac{\partial \mathcal{A}_{i j}^{\tau}}{\partial t}\right|, \quad\left|\frac{\partial \mathcal{A}_{i j}^{\tau}}{\partial s}\right| \leq C,
$$

for all $i, j=1, \ldots, N$ and for all relevant arguments. We denote

$$
a^{\varepsilon}(x, t)=a\left(x, t, \frac{x}{\varepsilon}\right) .
$$

We always assume that $A^{\tau}, a^{\varepsilon}$, are measurable in $\Omega_{T}$.

We let

$$
\alpha_{\sigma \tau}(t)=\alpha\left(\frac{1}{\sigma}\left\{\frac{t}{\tau}\right\}\right)
$$

where $\alpha \in \operatorname{Lip}(\boldsymbol{R})$ is a function such that

$$
\alpha(r) \geq 0, \quad \operatorname{supp} \alpha \subset(0,1), \quad\left|\alpha^{\prime}(r)\right| \leq C, \quad m_{\alpha}:=\int_{0}^{1} \alpha(r) \mathrm{d} r>0 .
$$

Thus supp $\alpha_{\sigma \tau} \subset A_{\sigma, \tau}$ and

$$
\left|\frac{\mathrm{d} \alpha_{\sigma \tau}}{\mathrm{d} t}\right| \leq C \frac{1}{\sigma \tau}
$$

Let $f \in L^{\infty}\left(\Omega_{T}\right)$ and let $u_{\tau}$ be the solution of the following parabolic problem

$$
\begin{aligned}
a^{\varepsilon}(x, t) \frac{\partial u_{\tau}}{\partial t}-\operatorname{div}\left(A^{\tau}(x, t) \nabla u_{\tau}\right) & =f, & & (x, t) \in \Omega_{T}^{\varepsilon}, \\
A^{\tau}(x, t) \nabla u_{\tau} \cdot \nu+\alpha_{\sigma \tau}(t) u_{\tau} & =0, & & (x, t) \in \Gamma^{\varepsilon} \times(0, T), \\
u_{\tau}(x, t) & =0, & & (x, t) \in \partial \Omega \times(0, T), \\
u_{\tau}(x, 0) & =u_{0}^{\tau}(x), & & x \in \Omega^{\varepsilon} .
\end{aligned}
$$

In this Section we assume all the needed smoothness of the solution $u_{\tau}$, whose existence is classical under standard regularity assumptions [21]. This can be achieved by means of a further approximation of the data and coefficients in the problem, keeping the structure required here. Specifically the source $f$ is approximated with smooth functions vanishing for small times. Thus the constants in our final estimates will not depend on $\tau, \varepsilon, \sigma$. 
The choice of the initial data $u_{0}^{\tau}$ deserves some clarification. We always assume that for a given $u_{0} \in L^{\infty}(\Omega)$

$u_{0}^{\tau} \rightarrow u_{0}, \quad$ strongly in $L^{2}(\Omega) ; \quad \int_{\Omega}\left|\nabla u_{0}^{\tau}\right|^{2} \mathrm{~d} x \leq \frac{\gamma}{\tau \varepsilon} ; \quad\left\|u_{0}^{\tau}\right\|_{L^{\infty}(\Omega)} \leq\left\|u_{0}\right\|_{L^{\infty}(\Omega)}$,

and that

the compatibility conditions of order 1 are satisfied on $\partial \Omega^{\varepsilon} \times\{0\}$;

see e.g., [21, Chapter IV].

Lemma 3.1. For any given $u_{0} \in L^{\infty}(\Omega)$ a sequence $\left\{u_{0}^{\tau}\right\}$ as in (3.10), (3.11) exists.

Proof. Given the data $u_{0} \in L^{\infty}(\Omega)$ we may construct a sequence $\left\{\widetilde{u_{0}^{\tau}}\right\} \subset C^{\infty}(\bar{\Omega})$ such that

$\widetilde{u_{0}^{\tau}} \rightarrow u_{0}, \quad$ strongly in $L^{2}(\Omega) ; \quad \int_{\Omega}\left|\nabla \widetilde{u_{0}^{\tau}}\right|^{2} \mathrm{~d} x \leq \frac{\gamma}{\tau \varepsilon} ; \quad\left\|\widetilde{u_{0}^{\tau}}\right\|_{L^{\infty}(\Omega)} \leq\left\|u_{0}\right\|_{L^{\infty}(\Omega)}$.

Next we introduce a sequence of cutoff functions $\left\{\eta^{\tau}\right\} \subset C^{\infty}(\bar{\Omega})$ such that

$$
\begin{gathered}
\eta^{\tau}(x)=1, \quad \operatorname{dist}\left(x, \Gamma^{\varepsilon} \cup \partial \Omega\right)>\tau ; \quad \eta^{\tau}(x)=0, \quad \operatorname{dist}\left(x, \Gamma^{\varepsilon} \cup \partial \Omega\right)<\tau / 2 ; \\
\left|\nabla \eta^{\tau}(x)\right| \leq \gamma \tau^{-1}, \quad x \in \Omega .
\end{gathered}
$$

We use here (3.1). We define

$$
u_{0}^{\tau}(x)=\widetilde{u_{0}^{\tau}}(x) \eta^{\tau}(x), \quad x \in \Omega .
$$

The motivation of this construction is threefold: First $u_{0}^{\tau}$ satisfies the compatibility conditions on $\Gamma^{\varepsilon}$ at $t=0$ required for the regularity of the solution. Second, recalling that $\left|\Gamma^{\varepsilon}\right| \leq \gamma / \varepsilon$, we have as $\tau \rightarrow 0$

$$
\begin{aligned}
& \int_{\Omega}\left|u_{0}-u_{0}^{\tau}\right|^{2} \mathrm{~d} x \leq \gamma \int_{\Omega}\left|u_{0}-\widetilde{u_{0}^{\tau}}\right|^{2} \mathrm{~d} x+\gamma\left\|u_{0}\right\|_{\infty}^{2} \int_{\Omega}\left(1-\eta^{\tau}\right)^{2} \mathrm{~d} x \\
& \leq \gamma \int_{\Omega}\left|u_{0}-\widetilde{u_{0}^{\tau}}\right|^{2} \mathrm{~d} x+\gamma\left\|u_{0}\right\|_{\infty}^{2}\left(\left|\Gamma^{\varepsilon}\right|+|\partial \Omega|\right) \tau \leq \gamma \int_{\Omega}\left|u_{0}-\widetilde{u_{0}^{\tau}}\right|^{2} \mathrm{~d} x+\gamma\left\|u_{0}\right\|_{\infty}^{2} \tau \varepsilon^{-1} \rightarrow 0 .
\end{aligned}
$$

Finally we have reasoning as in (3.12)

$$
\int_{\Omega}\left|\nabla u_{0}^{\tau}\right|^{2} \mathrm{~d} x \leq \gamma \int_{\Omega}\left\{\left(\eta^{\tau}\right)^{2}\left|\nabla \widetilde{u_{0}^{\tau}}\right|^{2}+\left(\widetilde{u_{0}^{\tau}}\right)^{2}\left|\nabla \eta^{\tau}\right|^{2}\right\} \mathrm{d} x \leq \gamma\left(\left\|u_{0}\right\|_{\infty}\right) \frac{1}{\tau \varepsilon} .
$$


As a consequence of the strong convergence $u_{0}^{\tau} \rightarrow u_{0}$ in $L^{2}(\Omega)$ one can easily prove for all $\phi \in L^{2}(\Omega)$

$$
\int_{\Omega^{\varepsilon}} u_{0}^{\tau}(x) \phi(x) \mathrm{d} x \rightarrow\left|Y^{*}\right| \int_{\Omega} u_{0}(x) \phi(x) .
$$

3.2. Estimates and convergence. A routine integration by parts and Gronwall's inequality prove the standard estimate

$$
\max _{0 \leq t \leq T} \int_{\Omega^{\varepsilon}} u_{\tau}^{2} \mathrm{~d} x+\int_{\Omega_{T}^{\varepsilon}}\left|\nabla u_{\tau}\right|^{2} \mathrm{~d} x \mathrm{~d} t+\int_{0}^{T} \int_{\Gamma^{\varepsilon}} \alpha_{\sigma \tau}(t) u_{\tau}^{2} \mathrm{~d} \sigma_{x} \mathrm{~d} t \leq \gamma .
$$

Proposition 3.2. The solution $u_{\tau}$ of problem (3.6)-(3.9) satisfies

$$
\left\|u_{\tau}\right\|_{L^{\infty}\left(\Omega_{T}^{\varepsilon}\right)} \leq M
$$

with $0<M:=\frac{\|f\|_{L^{\infty}\left(\Omega_{T}\right)}}{\min (a)} T+\left\|u_{0}\right\|_{L^{\infty}(\Omega)}$.

Proof. Let us here set for the sake of brevity

$$
\|f\|_{\infty}=\|f\|_{L^{\infty}\left(\Omega_{T}\right)}, \quad\left\|u_{0}\right\|_{\infty}=\left\|u_{0}\right\|_{L^{\infty}(\Omega)} .
$$

The function $w(x, t)=u_{\tau}(x, t)-\frac{\|f\|_{\infty}}{\min (a)} t-\left\|u_{0}\right\|_{\infty}$ satisfies

$$
\begin{cases}a^{\varepsilon} \frac{\partial w}{\partial t}-\operatorname{div}\left(A^{\tau} \nabla w\right)=f-a^{\varepsilon} \frac{\|f\|_{\infty}}{\min (a)}, & (x, t) \in \Omega_{T}^{\varepsilon}, \\ A \nabla w \cdot \nu+\alpha_{\sigma \tau}(t) w=-\alpha_{\sigma \tau}(t)\left(\frac{\|f\|_{\infty}}{\min (a)} t+\left\|u_{0}\right\|_{\infty}\right), & (x, t) \in \Gamma^{\varepsilon} \times(0, T), \\ w(x, t)=-\frac{\|f\|_{\infty}}{\min (a)} t-\left\|u_{0}\right\|_{\infty}, & (x, t) \in \partial \Omega \times(0, T), \\ w(x, 0)=u_{0}^{\tau}-\left\|u_{0}\right\|_{\infty}, & x \in \Omega^{\varepsilon}\end{cases}
$$

Then on multiplying the differential equation by the positive part $w_{+}$and integrating by parts in $\Omega_{T}^{\varepsilon}$ we obtain $w \leq 0$ and then $u_{\tau} \leq M$. The inequality $u_{\tau} \geq-M$ is proved in a similar way.

Proposition 3.3. We have the estimate

$$
\int_{\Omega_{T}^{\varepsilon}}\left(\frac{\partial u_{\tau}}{\partial t}\right)^{2} \mathrm{~d} x \mathrm{~d} t+\max _{0 \leq t \leq T} \int_{\Gamma^{\varepsilon}} \alpha_{\sigma \tau}(t) u_{\tau}(x, t)^{2} \mathrm{~d} \sigma+\max _{0 \leq t \leq T} \int_{\Omega^{\varepsilon}}\left|\nabla u_{\tau}(x, t)\right|^{2} \mathrm{~d} x \leq \frac{\gamma}{\tau \varepsilon} .
$$

Proof. We use $\frac{\partial u_{\tau}}{\partial t}$ as a test function for problem (3.6)-(3.9) and integrate by parts in the space variables in $\Omega_{T}^{\varepsilon}$, obtaining

$$
\int_{\Omega_{T}^{\varepsilon}} a^{\varepsilon}\left(\frac{\partial u_{\tau}}{\partial t}\right)^{2}+\int_{\Omega_{T}^{\varepsilon}} A^{\tau} \nabla u_{\tau} \cdot \nabla\left(\frac{\partial u_{\tau}}{\partial t}\right)-\int_{0}^{T} \int_{\Gamma^{\varepsilon}} A^{\tau} \nabla u_{\tau} \cdot \nu \frac{\partial u_{\tau}}{\partial t}=\int_{\Omega_{T}^{\varepsilon}} f \frac{\partial u_{\tau}}{\partial t} .
$$


Hence by an application of Cauchy-Schwarz inequality and integration by parts in time in the second integral in (3.18) we obtain

$$
\begin{aligned}
\int_{\Omega_{T}^{\varepsilon}}\left|\frac{\partial u_{\tau}}{\partial t}\right|^{2}+\int_{\Omega^{\varepsilon}}\left|\nabla u_{\tau}\right|^{2}(T) \leq-\gamma & \int_{0}^{T} \int_{\Gamma^{\varepsilon}} \alpha_{\sigma \tau} u_{\tau} \frac{\partial u_{\tau}}{\partial t} \\
& +\frac{\gamma}{\tau} \int_{\Omega_{T}^{\varepsilon}}\left|\nabla u_{\tau}\right|^{2}+\gamma \int_{\Omega_{T}^{\varepsilon}}|f|^{2}+\gamma \int_{\Omega^{\varepsilon}}\left|\nabla u_{0}^{\tau}\right|^{2}
\end{aligned}
$$

we have also made use of (3.3)-(3.4). The first integral on the right hand side of (3.19) can be estimated as follows

$$
\begin{aligned}
& \int_{0}^{T} \int_{\Gamma^{\varepsilon}} \alpha_{\sigma \tau} u_{\tau} \frac{\partial u_{\tau}}{\partial t}=\frac{1}{2} \int_{\Gamma^{\varepsilon}} \alpha_{\sigma \tau}(T) u_{\tau}^{2}(T)-\int_{0}^{T} \int_{\Gamma^{\varepsilon}} \frac{\mathrm{d} \alpha_{\sigma \tau}}{\mathrm{d} t} u_{\tau}^{2} \\
& \quad \geq \frac{1}{2} \int_{\Gamma^{\varepsilon}} \alpha_{\sigma \tau}(T) u_{\tau}^{2}(T)-M^{2} \int_{A_{\sigma, \tau}} \int_{\Gamma^{\varepsilon}} C(\sigma \tau)^{-1} \geq \frac{1}{2} \int_{\Gamma^{\varepsilon}} \alpha_{\sigma \tau}(T) u_{\tau}{ }^{2}(T)-\frac{\gamma}{\tau \varepsilon} .
\end{aligned}
$$

Here we have used $\left|A_{\sigma, \tau}\right| \leq \gamma \sigma$ and $\left|\Gamma^{\varepsilon}\right| \leq \gamma / \varepsilon$. Finally (3.17) follows when we collect (3.19)-(3.20), and use (3.15). Indeed, $T$ in the proof can be replaced with an arbitrary time.

Proposition 3.4. If $0<\delta<T / 2$ for any $0<h<\delta / 2$ we have

$$
\int_{\delta}^{T-\delta} \int_{\Omega^{\varepsilon}}\left|u_{\tau}(x, t+h)-u_{\tau}(x, t)\right|^{2} \mathrm{~d} x \mathrm{~d} t \leq \gamma\left(\sqrt{h}+\sqrt{\frac{\sigma}{\varepsilon}} h\right) .
$$

Here $\gamma$ depends on the constants in (3.15), (3.16) and on $\delta$.

Proof. Let $\delta \in(0, T / 2), 0<h<\delta / 2$, and define

$$
\varphi_{h}(x, t)=-\zeta(t) \int_{t}^{t+h} u_{\tau}(x, s) \mathrm{d} s
$$

where $\zeta \in C_{0}^{1}(\delta / 2, T-\delta / 2)$ is a nonnegative function such that $\zeta=1$ in $(\delta, T-\delta)$ and $\left|\zeta^{\prime}\right| \leq \gamma / \delta$. Here for any $v=v(x, t)$ we denote by $\widetilde{v}(x, t)=v(x, t+h)$ its time shift.

Testing equation (3.6) written at times $t$ and $t+h$ with $\varphi_{h}$ we get on subtracting 
the two integral formulations

$$
\begin{aligned}
-\int_{0} \int_{\Omega^{\varepsilon}}\left[\widetilde{a^{\varepsilon}} \widetilde{u_{\tau}}-a^{\varepsilon} u_{\tau}\right] \frac{\partial \varphi_{h}}{\partial t}-\int_{0}^{T} \int_{\Omega^{\varepsilon}}\left[\widetilde{a_{t}^{\varepsilon}} \widetilde{u_{\tau}}-a_{t}^{\varepsilon} u_{\tau}\right] \varphi_{h} \\
+\int_{0}^{T} \int_{\Omega^{\varepsilon}} \widetilde{A^{\tau}} \nabla \widetilde{u_{\tau}} \cdot \nabla \varphi_{h}-\int_{0}^{T} \int_{\Omega^{\varepsilon}} A^{\tau} \nabla u_{\tau} \cdot \nabla \varphi_{h}= \\
\\
\int_{0}^{T} \int_{\Gamma^{\varepsilon}}\left[\widetilde{\alpha_{\sigma \tau}} \widetilde{u_{\tau}}-\alpha_{\sigma \tau} u_{\tau}\right] \varphi_{h}+\int_{0}^{T} \int_{\Omega^{\varepsilon}}[\widetilde{f}-f] \varphi_{h} .
\end{aligned}
$$

The first integral on the left hand side of (3.22) equals

$$
\begin{aligned}
\int_{0}^{T} \int_{\Omega^{\varepsilon}} & {\left[\widetilde{a^{\varepsilon}} \widetilde{u_{\tau}}-a^{\varepsilon} u_{\tau}\right]\left\{\zeta^{\prime} \int_{t}^{t+h} u_{\tau}(x, s) \mathrm{d} s+\zeta\left[\widetilde{u_{\tau}}-u_{\tau}\right]\right\}=\int_{0} \int_{\Omega^{\varepsilon}}\left[\widetilde{u_{\tau}}-u_{\tau}\right]^{2} \zeta a^{\varepsilon} } \\
& +\int_{0}^{T} \int_{\Omega^{\varepsilon}}\left[\widetilde{a^{\varepsilon}} \widetilde{u_{\tau}}-a^{\varepsilon} u_{\tau}\right] \zeta^{\prime} \int_{t}^{t+h} u_{\tau}(x, s) \mathrm{d} s+\int_{0}^{T} \int_{\Omega^{\varepsilon}} \widetilde{u_{\tau}}\left[\widetilde{u_{\tau}}-u_{\tau}\right] \zeta\left[\widetilde{a^{\varepsilon}}-a^{\varepsilon}\right] .
\end{aligned}
$$

The first term on the right hand side of (3.23) essentially is the one estimated in the statement. The second integral there can be majorized by means of Hölder inequality by

$$
\begin{aligned}
\gamma\left\|\zeta^{\prime}\right\|_{\infty}\left(\int_{\Omega^{\varepsilon}} \int_{\delta / 2}^{T-\delta / 2}\left|\widetilde{u_{\tau}}\right|^{2}+\left|u_{\tau}\right|^{2}\right)^{\frac{1}{2}}\left(\int_{\Omega^{\varepsilon}} \int_{\delta / 2}^{T-\delta / 2}\left|\int_{t}^{t+h} u_{\tau}(x, s) \mathrm{d} s\right|^{2}\right)^{\frac{1}{2}} & \leq \gamma\left\|u_{\tau}\right\|_{L^{2}\left(\Omega_{T}^{\varepsilon}\right)}^{2} \sqrt{h} .
\end{aligned}
$$

The third term on the right hand side of (3.23) is estimated, invoking the time regularity of $a$, by

$$
\gamma \int_{\Omega^{\varepsilon}} \int_{\delta / 2}^{T-\delta / 2}\left|\widetilde{u_{\tau}}\right|\left(\left|\widetilde{u_{\tau}}\right|+\left|u_{\tau}\right|\right)\left|\widetilde{a^{\varepsilon}}-a^{\varepsilon}\right| \leq \gamma\left\|u_{\tau}\right\|_{L^{2}\left(\Omega_{T}^{\varepsilon}\right)}^{2} h .
$$

The other integrals over $\Omega_{T}^{\varepsilon}$ appearing in (3.22) can be estimated by

$$
\gamma\left(\left\|u_{\tau}\right\|_{L^{2}\left(\Omega_{T}^{\varepsilon}\right)}^{2}+\left\|\nabla u_{\tau}\right\|_{L^{2}\left(\Omega_{T}^{\varepsilon}\right)}^{2}+\|f\|_{L^{2}\left(\Omega_{T}^{\varepsilon}\right)}^{2}\right) \sqrt{h} .
$$

This follows from routine reasoning, but see however [3]. We deal in greater detail with the boundary integral in (3.22). We use the maximum principle for $u_{\tau}$, 
obtaining

$$
\begin{aligned}
\left|\int_{0} \int_{\Gamma^{\varepsilon}}\left[\widetilde{\alpha_{\sigma \tau}} \widetilde{u_{\tau}}-\alpha_{\sigma \tau} u_{\tau}\right] \varphi_{h}\right| \leq & \gamma\left(\int_{0}^{T-\delta / 2} \int_{\Gamma^{\varepsilon}}\left|\sqrt{\widetilde{\alpha_{\sigma \tau}}} \widetilde{u_{\tau}}\right|^{2}+\left|\sqrt{\alpha_{\sigma \tau}} u_{\tau}\right|^{2}\right)^{\frac{1}{2}} \\
& \times\left(\int_{0}^{T-\delta / 2} \int_{\Gamma^{\varepsilon}}\left|\left[\sqrt{\widetilde{\alpha_{\sigma \tau}}}+\sqrt{\alpha_{\sigma \tau}}\right] \int_{t}^{t+h}\right| u_{\tau}(x, s)|\mathrm{d} s|^{2}\right)^{\frac{1}{2}} \\
\leq & \gamma\left\|\sqrt{\alpha_{\sigma \tau}} u_{\tau}\right\|_{L^{2}\left(\Gamma^{\varepsilon} \times(0, T)\right)} M(\sigma)^{\frac{1}{2}}\left|\Gamma^{\varepsilon}\right|^{\frac{1}{2}} h .
\end{aligned}
$$

We have used the fact that $\left|A_{\sigma, \tau}\right| \leq \gamma \sigma$. Finally recalling that $\left|\Gamma^{\varepsilon}\right| \leq \gamma / \varepsilon$ and collecting all the estimates above we get (3.21).

Remark 3.5. By using estimates (3.15) and (3.21) we can prove the strong convergence of $\mathcal{T}_{\tau}^{*}\left(u_{\tau}\right)$ in $L^{2}\left(\Omega_{T} \times Q^{*}\right)$, up to a subsequence.

Lemma 3.6. There exists an extension $\bar{u}_{\tau} \in L^{2}\left(0, T ; W^{1,2}(\Omega)\right)$ of $u_{\tau}$, and a $\bar{u} \in$ $L^{2}\left(0, T ; W^{1,2}(\Omega)\right)$ such that, up to a subsequence,

$$
\begin{aligned}
\bar{u}_{\tau} & \rightarrow \bar{u}, & & \text { strongly in } L^{2}\left(\Omega_{T}\right), \\
\nabla \bar{u}_{\tau} & \rightarrow \nabla \bar{u}, & & \text { weakly in } L^{2}\left(\Omega_{T}\right) .
\end{aligned}
$$

Proof. We may use the linear and continuous extension operator $\mathcal{P}$ as in the proof of [15, Lemma 2.1]; the extension $\bar{u}_{\tau}(t)=\mathcal{P}\left(u_{\tau}(t)\right)$ of $u_{\tau}$ in $\Omega$ satisfies the estimates

$$
\begin{gathered}
\left\|\mathcal{P}\left(u_{\tau}(t)\right)\right\|_{L^{2}(\Omega)}=\left\|\bar{u}_{\tau}(t)\right\|_{L^{2}(\Omega)} \leq \gamma\left\|u_{\tau}(t)\right\|_{L^{2}\left(\Omega^{\varepsilon}\right)}, \\
\left\|\nabla\left(\mathcal{P}\left(u_{\tau}(t)\right)\right)\right\|_{L^{2}(\Omega)}=\left\|\nabla \bar{u}_{\tau}(t)\right\|_{L^{2}(\Omega)} \leq \gamma\left\|\nabla u_{\tau}(t)\right\|_{L^{2}\left(\Omega^{\varepsilon}\right)},
\end{gathered}
$$

where $\gamma$ is a constant independent of $\varepsilon$. Time compactness for $\bar{u}_{\tau}$ follows from the estimates above, the linearity of the extension operator $\mathcal{P}$ and Proposition 3.4. Indeed, using the same notation as therein, we get

$$
\begin{aligned}
\int_{\delta}^{T-\delta} \int_{\Omega}\left|\mathcal{P}\left(u_{\tau}\right)(t+h)-\mathcal{P}\left(u_{\tau}\right)(t)\right|^{2} & =\int_{\delta}^{T-\delta} \int_{\Omega}\left|\mathcal{P}\left(u_{\tau}(t+h)-u_{\tau}(t)\right)\right|^{2} \\
& \leq \gamma \int_{\delta}^{T-\delta} \int_{\Omega^{\varepsilon}}\left|u_{\tau}(t+h)-u_{\tau}(t)\right|^{2} \leq \gamma\left(\sqrt{h}+\sqrt{\frac{\sigma}{\varepsilon}} h\right) .
\end{aligned}
$$

Then by the standard theory of Sobolev spaces we get the strong convergence of $\bar{u}_{\tau}$ in $L^{2}\left(\Omega_{T}\right)$, by extracting a subsequence if necessary. The weak convergence of the space gradients follows simply by the boundedness of $\mathcal{P}$ and by (3.15).

The introduction of the extension operator is motivated by the following 
Proposition 3.7. We may extract a subsequence of $u_{\tau}$ such that

$$
\mathcal{T}_{\tau}^{*}\left(u_{\tau}\right) \rightarrow u, \quad \text { weakly in } L^{2}\left(\Omega_{T}, W^{1,2}\left(Q^{*}\right)\right)
$$

and $u \in L^{2}\left(0, T ; W^{1,2}(\Omega)\right)$.

Proof. Convergence (3.29) is a consequence of Proposition 2.14 with $\theta_{\tau}=1 / \sqrt{\tau \varepsilon}$ applied to $u_{\tau}$. To prove that $u \in L^{2}\left(0, T ; W^{1,2}(\Omega)\right)$, we consider an extension of $u_{\tau}$ to $\Omega_{T}$ as in Lemma 3.6, so that invoking first (3.29) we have

$$
\begin{aligned}
u=\text { weak } L^{2}-\lim _{\tau \rightarrow 0} \mathcal{T}_{\tau}^{*}\left(u_{\tau}\right)= & \text { weak } L^{2}-\lim _{\tau \rightarrow 0} \mathcal{T}_{\tau}\left(\bar{u}_{\tau}\right)_{\mid \Omega_{T} \times Q^{*}} \\
& =\text { strong } L^{2}-\lim _{\tau \rightarrow 0} \mathcal{T}_{\tau}\left(\bar{u}_{\tau}\right)_{\mid \Omega_{T} \times Q^{*}}=\bar{u}_{\mid \Omega_{T} \times Q^{*}}=\bar{u} .
\end{aligned}
$$

The last two equalities in (3.30) are a consequence of Proposition 2.11, which implies that $\bar{u}=\bar{u}(x, t)$. Here we have used the strong convergence of $\bar{u}_{\tau}$, so that we may apply $(2.15)$ to infer that the limit of $\mathcal{T}_{\tau}\left(\bar{u}_{\tau}\right)_{\mid \Omega_{T} \times Q^{*}}$ can be actually taken in the strong sense. We conclude that $u=\bar{u} \in L^{2}\left(0, T ; W^{1,2}(\Omega)\right)$.

Remark 3.8. The result in Proposition 3.7 allows us to apply Theorems 2.21 and 2.24 to $u_{\tau}$.

\section{The limit PRoblem in the CASE $\sigma \leq \gamma \varepsilon$}

Recall that by virtue of Proposition 3.3 we may choose $\theta_{\tau}=\frac{1}{\sqrt{\tau \varepsilon}}$, so that $(2.19)$ and (2.21) are satisfied owing to (3.1). In this section we assume

$$
\lim _{\varepsilon \rightarrow 0} \frac{\sigma}{\varepsilon}=\beta \in[0, \infty),
$$

and consider two different subcases.

4.1. The case of fast oscillations. We consider here the case when

$$
\frac{\tau \theta_{\tau}}{\varepsilon}=\left(\frac{\tau}{\varepsilon^{3}}\right)^{\frac{1}{2}} \leq C
$$

Assume throughout that there exist bounded functions $A^{0}: \Omega_{T} \times Q^{*} \rightarrow \boldsymbol{R}^{N^{2}}$, $b: \Omega_{T} \times Y^{*} \rightarrow \boldsymbol{R}$ such that

$$
\begin{array}{cl}
\mathcal{T}_{\tau}^{*}\left(A^{\tau}\right) \rightarrow A^{0}, & \text { strongly in } L^{1}\left(\Omega_{T} \times Q^{*}\right), \\
\mathcal{T}_{\tau}^{*}\left(a^{\varepsilon}\right) \rightarrow b, & \text { strongly in } L^{1}\left(\Omega_{T} \times Y^{*}\right) .
\end{array}
$$

We also need to assume

$$
\mathcal{T}_{\tau}^{*}\left(a_{t}^{\varepsilon}\right) \rightarrow b_{t}, \quad \text { strongly in } L^{1}\left(\Omega_{T} \times Y^{*}\right),
$$

and the convergence at time $t=0$

$$
\mathcal{T}_{\tau}^{*}\left(a^{\varepsilon}(0)\right) \rightarrow b(0), \quad \text { weakly in } L^{2}\left(\Omega \times Y^{*}\right) .
$$


Remark 4.1. Actually the only classes of functions for which the convergences (4.3)-(4.4) are known to hold in this context, are sums of the following cases: $\phi=f_{1}(x, t) f_{2}(y, s), \phi \in L^{p}\left(Q ; C\left(\Omega_{T}\right)\right), \phi \in L^{p}\left(\Omega_{T} ; C(Q)\right)$. In all such cases $\mathcal{T}_{\tau}^{*}\left(\phi^{\tau}\right) \rightarrow \phi$ strongly in $L^{p}\left(\Omega_{T} \times Q\right)$. See $[2,11,13]$. The other requirements obey a similar remark.

Theorem 4.2. Let (4.1)-(4.6) hold true. Let $u_{\tau}$ be the solution of problem (3.6)(3.9); then there exist $u \in L^{2}\left(0, T, W^{1,2}(\Omega)\right)$ and $\widehat{u} \in L^{2}\left(\Omega_{T}, W_{\text {per }}^{1,2}\left(Y^{*}\right)\right)$ so that $\mathcal{M}_{Y^{*}}(\widehat{u})=0$ and

$$
\begin{aligned}
\mathcal{T}_{\tau}^{*}\left(u_{\tau}\right) & \rightarrow u, & & \text { weakly in } L^{2}\left(\Omega_{T}, W^{1,2}\left(Q^{*}\right)\right), \\
\mathcal{T}_{\tau}^{*}\left(\nabla u_{\tau}\right) & \rightarrow \nabla u+\nabla_{y} \widehat{u}, & & \text { weakly in } L^{2}\left(\Omega_{T} \times Q^{*}\right), \\
\frac{\tau}{\varepsilon} \mathcal{T}_{\tau}^{*}\left(\frac{\partial u_{\tau}}{\partial t}\right) & \rightarrow 0, & & \text { weakly in } L^{2}\left(\Omega_{T} \times Q^{*}\right) .
\end{aligned}
$$

The pair $(u, \widehat{u})$ is the solution of the problem

$$
\begin{array}{r}
\frac{1}{\left|Y^{*}\right|} \int_{\Omega_{T}} \int_{Y^{*}}\left\{-u(b \varphi)_{t}+\mathcal{M}_{\Sigma}\left(A^{0}\right)\left(\nabla_{x} u+\nabla_{y} \widehat{u}\right) \cdot\left(\nabla_{x} \varphi+\nabla_{y} \Psi\right)\right\} \mathrm{d} x \mathrm{~d} t \mathrm{~d} y+ \\
\beta m_{\alpha} \frac{|\partial B|}{\left|Y^{*}\right|} \int_{\Omega_{T}} u \varphi \mathrm{d} x \mathrm{~d} t=\int_{\Omega_{T}} f \varphi \mathrm{d} x \mathrm{~d} t+\int_{\Omega} b(x, 0) u_{0}(x) \varphi(x, 0) \mathrm{d} x,
\end{array}
$$

for all $\varphi \in W^{1,2}\left(\Omega_{T}\right)$ with $\varphi=0$ on $\partial \Omega \times(0, T)$ and on $t=T$, and $\Psi \in$ $L^{2}\left(\Omega_{T}, W_{\text {per }}^{1,2}\left(Y^{*}\right)\right)$.

Proof. Here we may assume in any case that $u$ is the limit function introduced in Proposition 3.7, so that (4.7) holds true, at least for a subsequence.

If $\tau / \varepsilon^{3} \rightarrow 0$, on recalling the estimate (3.17) and Remark 3.8 we may apply Theorem 2.24. In this way we infer the existence of $\widehat{u}$ as in $(2.42)-(2.44)$, so that $\widehat{u}=\widehat{u}(x, t, y)$.

If $\tau / \varepsilon^{3} \leq C$ so that (2.40) holds true, we use Theorem 2.24 to obtain a function $\widehat{u}$ still satisfying (2.42) and (2.43). In order to show that $\widehat{u}$ is independent of $s$ even in this case, we reason as follows. We test equation (3.6) with the function

$$
\frac{\tau}{\varepsilon} \varphi(x, t) \psi\left(\frac{x}{\varepsilon}, \frac{t}{\tau}\right)
$$

where $\varphi \in \mathcal{C}^{\infty}\left(\overline{\Omega_{T}}\right)$ with the support of $\varphi$ bounded away from $\partial \Omega \times[0, T]$ and $\Omega \times\{T\}$, and $\psi \in W_{\text {per }}^{1,2}\left(Q^{*}\right)$. We calculate

$$
\begin{array}{r}
\frac{\tau}{\varepsilon} \int_{\Omega_{T}^{\varepsilon}} a^{\varepsilon} \frac{\partial u_{\tau}}{\partial t} \varphi \psi \mathrm{d} x \mathrm{~d} t+\frac{\tau}{\varepsilon} \int_{\Omega_{T}^{\varepsilon}} A^{\tau} \nabla u_{\tau} \cdot(\nabla \varphi) \psi \mathrm{d} x \mathrm{~d} t+\frac{\tau}{\varepsilon^{2}} \int_{\Omega_{T}^{\varepsilon}} A^{\tau} \nabla u_{\tau}\left(\nabla_{y} \psi\right) \varphi \mathrm{d} x \mathrm{~d} t \\
+\frac{\tau}{\varepsilon} \int_{\Gamma_{T}^{\varepsilon}} \alpha_{\sigma \tau} u_{\tau} \varphi \psi \mathrm{d} \sigma \mathrm{d} t=\frac{\tau}{\varepsilon} \iint_{\Omega_{T}^{\varepsilon}} f \varphi \psi \mathrm{d} x \mathrm{~d} t
\end{array}
$$


Clearly, with the exception of the first one, all the terms in (4.11) are vanishingly small as $\tau \rightarrow 0$; we remark explicitly that the last term on the left hand side is bounded by $\gamma \tau / \varepsilon^{2}$. On unfolding the first integral we therefore get

$$
\frac{\tau}{\varepsilon} \int_{\Omega_{T}} \int_{Q^{*}} \mathcal{T}_{\tau}^{*}\left(a^{\varepsilon}\right) \mathcal{T}_{\tau}^{*}\left(\frac{\partial u_{\tau}}{\partial t}\right) \mathcal{T}_{\tau}^{*}(\varphi) \mathcal{T}_{\tau}^{*}(\psi) \mathrm{d} x \mathrm{~d} t \mathrm{~d} y \mathrm{~d} s \rightarrow 0 .
$$

Using (2.43) we obtain in the limit exactly $\frac{\partial \widehat{u}}{\partial s}=0$.

Next we use $\varphi$ as above as a test function for problem (3.6)-(3.9). Integrating by parts in $\Omega_{T}^{\varepsilon}$ we get

$$
\begin{aligned}
-\int_{\Omega_{T}^{\varepsilon}} a^{\varepsilon} u_{\tau} \varphi_{t} \mathrm{~d} x \mathrm{~d} t-\int_{\Omega_{T}^{\varepsilon}} a_{t}^{\varepsilon} u_{\tau} \varphi \mathrm{d} x \mathrm{~d} t+\int_{\Omega_{T}^{\varepsilon}} A^{\tau} \nabla u_{\tau} \cdot \nabla \varphi \mathrm{d} x \mathrm{~d} t \\
\quad+\int_{\Gamma_{T}^{\varepsilon}} \alpha_{\sigma \tau} u_{\tau} \varphi \mathrm{d} \sigma \mathrm{d} t=\int_{\Omega_{T}^{\varepsilon}} f \varphi \mathrm{d} x \mathrm{~d} t+\int_{\Omega^{\varepsilon}} a^{\varepsilon}(x, 0) u_{0}^{\tau}(x) \varphi(x, 0) \mathrm{d} x .
\end{aligned}
$$

Unfolding we get

$$
\begin{gathered}
-\int_{\Omega_{T}} \int_{Q^{*}} \mathcal{T}_{\tau}^{*}\left(a^{\varepsilon}\right) \mathcal{T}_{\tau}^{*}\left(u_{\tau}\right) \mathcal{T}_{\tau}^{*}\left(\varphi_{t}\right) \mathrm{d} x \mathrm{~d} t \mathrm{~d} y \mathrm{~d} s-\int_{\Omega_{T}} \int_{Q^{*}} \mathcal{T}_{\tau}^{*}\left(a_{t}^{\varepsilon}\right) \mathcal{T}_{\tau}^{*}\left(u_{\tau}\right) \mathcal{T}_{\tau}^{*}(\varphi) \mathrm{d} x \mathrm{~d} t \mathrm{~d} y \mathrm{~d} s \\
\quad+\int_{\Omega_{T}} \int_{Q^{*}} \mathcal{T}_{\tau}^{*}\left(A^{\tau}\right) \mathcal{T}_{\tau}^{*}\left(\nabla u_{\tau}\right) \mathcal{T}_{\tau}^{*}(\nabla \varphi) \mathrm{d} x \mathrm{~d} t \mathrm{~d} y \mathrm{~d} s \\
+\frac{\sigma}{\varepsilon} \int_{\Omega_{T}} \int_{\partial B} \int_{\Sigma} \mathcal{T}_{\tau, \sigma}^{b}\left(\alpha_{\sigma \tau}\right) \mathcal{T}_{\tau, \sigma}^{b}\left(u_{\tau}\right) \mathcal{T}_{\tau, \sigma}^{b}(\varphi) \mathrm{d} x \mathrm{~d} t \mathrm{~d} \sigma_{y} \mathrm{~d} r=\int_{\Omega_{T}^{\varepsilon}} f \varphi \mathrm{d} x \mathrm{~d} t \\
+\int_{\Omega} \int_{Y^{*}} \mathcal{T}_{\tau}^{*}\left(a^{\varepsilon}(x, 0)\right) \mathcal{T}_{\tau}^{*}\left(u_{0}^{\tau}(x)\right) \mathcal{T}_{\tau}^{*}(\varphi(x, 0)) \mathrm{d} x \mathrm{~d} y+R^{\tau},
\end{gathered}
$$

where $R^{\tau}=o(1)$, as $\tau \rightarrow 0$. Notice that the last integral on the left hand side of (4.14) is in principle calculated over the domain $\Sigma / \sigma$ of the ultra-micro time variable. However as a matter of fact, $\mathcal{T}_{\tau, \sigma}^{b}\left(\alpha_{\sigma \tau}\right)=0$ outside of $\Sigma$, owing to the definition of $\alpha_{\sigma \tau}$.

Then, in the case $\beta>0$, we take the limit $\tau \rightarrow 0$, considering the strong convergence of $u_{0}^{\tau}$ and also exploiting Proposition 2.14, as well as Proposition 2.20. Indeed in the case $\beta>0$ (2.35) with $p=2$ is clearly fulfilled. Thus we get

$$
\begin{aligned}
& -\int_{\Omega_{T}} \int_{Q^{*}} u(b \varphi)_{t} \mathrm{~d} x \mathrm{~d} t \mathrm{~d} y \mathrm{~d} s+\int_{\Omega_{T}} \int_{Q^{*}} A^{0}\left(\nabla_{x} u+\nabla_{y} \widehat{u}\right) \cdot \nabla \varphi \mathrm{d} x \mathrm{~d} t \mathrm{~d} y \mathrm{~d} s \\
& +\beta|\partial B| \int_{\Omega_{T}} \int_{\Sigma} \alpha(r) u \varphi \mathrm{d} x \mathrm{~d} t \mathrm{~d} r=\left|Y^{*}\right| \int_{\Omega_{T}} f \varphi \mathrm{d} x \mathrm{~d} t+\left|Y^{*}\right| \int_{\Omega} b(x, 0) u_{0}(x) \varphi(x, 0) \mathrm{d} x .
\end{aligned}
$$


We have used also Proposition 2.13 for $\varphi$. If $\beta=0$ we obtain the same result since the integral multiplied by $\sigma / \varepsilon$ in (4.14) is uniformly bounded. The macroscopic part of (4.10) follows when we note that all the functions in (4.15) are independent of $s$ and $r$, excepting $A^{0}$ and $\alpha(r)$, and of course we divide by $\left|Y^{*}\right|$. By density the just proved integral equation holds for $\varphi \in W^{1,2}\left(\Omega_{T}\right)$ vanishing on $\partial \Omega$ and for $t=T$.

Finally in order to prove the microscopic part of (4.10) we use as a test function

$$
\varepsilon \varphi(x, t) \psi\left(\frac{x}{\varepsilon}\right)
$$

with $\varphi$ as above and $\psi \in W_{p e r}^{1,2}\left(Y^{*}\right)$. We obtain

$$
\begin{gathered}
-\varepsilon \int_{\Omega_{T}^{\varepsilon}} u_{\tau}\left(a^{\varepsilon} \varphi\right)_{t} \psi+\varepsilon \int_{\Omega_{T}^{\varepsilon}} A^{\tau} \nabla u_{\tau} \cdot(\nabla \varphi) \psi+\int_{\Omega_{T}^{\varepsilon}} A^{\tau} \nabla u_{\tau} \cdot\left(\nabla_{y} \psi\right) \varphi \\
\quad+\varepsilon \int_{\Gamma_{T}^{\varepsilon}} \alpha_{\sigma \tau} u_{\tau} \varphi \psi=\varepsilon \int_{\Omega_{T}^{\varepsilon}} f \varphi \psi+\varepsilon \int_{\Omega^{\varepsilon}} a^{\varepsilon}(x, 0) u_{0}^{\tau}(x) \varphi(x, 0) \psi\left(\frac{x}{\varepsilon}\right) .
\end{gathered}
$$

All the terms in (4.16) clearly tend to 0 , with the possible exception of the third and fourth ones on the left hand side. These can be unfolded leading us to

$$
\begin{aligned}
\int_{\Omega_{T}} \int_{Q^{*}} \mathcal{T}_{\tau}^{*} & \left(A^{\tau}\right) \mathcal{T}_{\tau}^{*}\left(\nabla u_{\tau}\right) \mathcal{T}_{\tau}^{*}\left(\nabla_{y} \psi\right) \mathcal{T}_{\tau}^{*}(\varphi) \mathrm{d} x \mathrm{~d} t \mathrm{~d} y \mathrm{~d} s \\
& +\varepsilon \frac{\sigma}{\varepsilon} \int_{\Omega_{T}} \int_{\partial B} \int_{\Sigma} \mathcal{T}_{\tau, \sigma}^{b}\left(\alpha_{\sigma \tau}\right) \mathcal{T}_{\tau, \sigma}^{b}\left(u_{\tau}\right) \mathcal{T}_{\tau, \sigma}^{b}(\varphi) \mathcal{T}_{\tau, \sigma}^{b}(\psi) \mathrm{d} x \mathrm{~d} t \mathrm{~d} \sigma_{y} \mathrm{~d} r=R^{\tau} .
\end{aligned}
$$

We apply again (2.42) to the first term in (4.17), while it is now clear that the second one there vanishes in the limit. Hence we conclude

$$
\int_{\Omega_{T}} \int_{Y^{*}} \mathcal{M}_{\Sigma}\left(A^{0}\right)\left(\nabla u+\nabla_{y} \widehat{u}\right) \cdot\left(\nabla_{y} \psi\right) \varphi \mathrm{d} x \mathrm{~d} t \mathrm{~d} y=0 .
$$

On invoking the density of the tensor product $\mathcal{C}^{\infty}\left(\overline{\Omega_{T}}\right) \otimes W_{\text {per }}^{1,2}\left(Y^{*}\right)$ in $L^{2}\left(\Omega_{T}, W_{\text {per }}^{1,2}\left(Y^{*}\right)\right)$ we see that (4.18) holds for every $\Psi \in L^{2}\left(\Omega_{T}, W_{\text {per }}^{1,2}\left(Y^{*}\right)\right)$.

Uniqueness of solution can be proven by means of standard arguments, exploiting the linearity of the problem (see Theorem 4.2 in [3]). Notice that uniqueness implies the convergence of the whole sequence $u_{\tau}$.

Next we give a more precise formulation of (4.10). Let us denote the elements of the limit matrix in (4.3) by

$$
A^{0}(x, t, y, s)=\left(b_{i, j}(x, t, y, s)\right)_{1 \leq i, j \leq N},
$$

then, as usual in literature, we factorize

$$
\widehat{u}(x, t, y)=-\nabla_{x} u(x, t) \cdot \sum_{i=1}^{N} \chi_{i}(x, t, y) e_{i}, \quad(x, t, y) \in \Omega_{T} \times Y^{*},
$$


where $\chi_{i}$ for $1 \leq i \leq N$ satisfy $\mathcal{M}_{Y^{*}}\left(\chi_{i}\right)=0$. By using (4.19) in (4.10) with $\varphi=0$ we find that $\chi_{i}$ are the $Y$-periodic solutions of the problem

$$
\begin{aligned}
& \sum_{j, k=1}^{N} \frac{\partial}{\partial y_{j}}\left(\mathcal{M}_{\Sigma}\left(b_{j, k}\right)(x, t, y) \frac{\partial\left(\chi_{i}-y_{i}\right)}{\partial y_{k}}\right)=0 \quad \text { in } \Omega_{T} \times Y^{*} \\
& \sum_{j, k=1}^{N} \mathcal{M}_{\Sigma}\left(b_{j, k}\right)(x, t, y) \frac{\partial\left(\chi_{i}-y_{i}\right)}{\partial y_{k}} \cdot \nu=0 \quad \text { on } \Omega_{T} \times \partial B \\
& \chi_{i} \text { are } Y \text {-periodic. }
\end{aligned}
$$

Then considering (4.10) with $\Psi=0$ and using again (4.19), we find that $u$ solves

$$
\begin{aligned}
\mathcal{M}_{Y^{*}}(b) u_{t}-\operatorname{div}\left(\mathcal { M } _ { Y ^ { * } } \left(\mathcal{M}_{\Sigma}\left(A^{0}\right)(I\right.\right. & \left.\left.\left.-\left[\nabla_{y} \chi_{1}|\cdots| \nabla_{y} \chi_{N}\right]\right)\right) \nabla u\right) \\
& +\beta m_{\alpha} \frac{|\partial B|}{\left|Y^{*}\right|} u=f, \quad(x, t) \in \Omega_{T},
\end{aligned}
$$

and

$$
\begin{array}{rlr}
u(x, t) & =0, & (x, t) \in \partial \Omega \times(0, T), \\
u(x, 0) & =u_{0}, & x \in \Omega .
\end{array}
$$

4.2. The case of slow oscillations. We consider here the case when (4.2) does not hold. Clearly we may assume

$$
\lim _{\tau \rightarrow 0} \frac{\tau}{\varepsilon^{3}}=\infty .
$$

Even if $\tau / \varepsilon^{3}$ does not stay bounded in the limit we can still get a limit problem, but we need to restrict ourselves to the case

$$
A^{\tau}(x, t)=\mathcal{A}^{\tau}\left(x, t, \frac{x}{\varepsilon}\right), \quad \mathcal{A}^{\tau}=\mathcal{A}^{\tau}(x, t, y) .
$$

We also assume that there exist bounded functions $A^{0}: \Omega_{T} \times Y^{*} \rightarrow \boldsymbol{R}^{N^{2}}, b$ : $\Omega_{T} \times Y^{*} \rightarrow \boldsymbol{R}$ such that (4.3)-(4.6) hold true. The main difference with respect to the case studied in Subsection 4.1 is that the microscopic function $\widetilde{u}$ does depend on $s$ in this setting. See Remarks 4.5 and 4.6.

Theorem 4.3. Let (4.1), (4.3)-(4.6) and (4.26) hold true. Let $u_{\tau}$ be the solution of problem (3.6)-(3.9). Then there exist $u \in L^{2}\left(0, T, W^{1,2}(\Omega)\right)$ and $\widetilde{u} \in L^{2}\left(\Omega_{T} \times\right.$ $\left.\Sigma, W_{\text {per }}^{1,2}\left(Y^{*}\right)\right)$ with $\mathcal{M}_{Y^{*}}(\widetilde{u})=0$ so that up to subsequences

$$
\begin{aligned}
\mathcal{T}_{\tau}^{*}\left(u_{\tau}\right) & \rightarrow u, & & \text { weakly in } L^{2}\left(\Omega_{T}, W^{1,2}\left(Q^{*}\right)\right), \\
\mathcal{T}_{\tau}^{*}\left(\nabla u_{\tau}\right) & \rightarrow \nabla u+\nabla_{y} \tilde{u}, & & \text { weakly in } L^{2}\left(\Omega_{T} \times Q^{*}\right) .
\end{aligned}
$$


If we define $\widetilde{u}_{\Sigma}(x, t, y)=\mathcal{M}_{\Sigma}(\widetilde{u})(x, t, y)$, the pair $\left(u, \widetilde{u}_{\Sigma}\right)$ is the solution of the problem

$$
\begin{aligned}
& \frac{1}{\left|Y^{*}\right|} \int_{\Omega_{T}} \int_{Y^{*}}\left\{-u(b \varphi)_{t}+A^{0}\left(\nabla_{x} u+\nabla_{y} \widetilde{u}_{\Sigma}\right) \cdot\left(\nabla_{x} \varphi+\nabla_{y} \Psi\right)\right\} \mathrm{d} x \mathrm{~d} t \mathrm{~d} y \\
& \quad+\beta m_{\alpha} \frac{|\partial B|}{\left|Y^{*}\right|} \int_{\Omega_{T}} u \varphi \mathrm{d} x \mathrm{~d} t=\int_{\Omega_{T}} f \varphi \mathrm{d} x \mathrm{~d} t+\int_{\Omega} b(x, 0) u_{0}(x) \varphi(x, 0) \mathrm{d} x,
\end{aligned}
$$

for $\varphi \in W^{1,2}\left(\Omega_{T}\right)$ with $\varphi=0$ on $\partial \Omega \times(0, T), \varphi(x, T)=0$ for $x \in \Omega$, and $\Psi \in L^{2}\left(\Omega_{T}, W_{\text {per }}^{1,2}\left(Y^{*}\right)\right)$.

Proof. Here we may assume that $u$ is the limit function introduced in Proposition 3.7 with $\theta_{\tau}=1 / \sqrt{\tau \varepsilon}$, so that (4.28) holds true. We may apply Theorem 2.21 in order to obtain (4.29).

Next we reason as in the part of the proof of Theorem 4.2 following (4.13), we obtain

$$
\begin{gathered}
-\int_{\Omega_{T}} \int_{Q^{*}} u(b \varphi)_{t} \mathrm{~d} x \mathrm{~d} t \mathrm{~d} y \mathrm{~d} s+\int_{\Omega_{T}} \int_{Q^{*}} A^{0}\left(\nabla u+\nabla_{y} \widetilde{u}\right) \cdot \nabla \varphi \mathrm{d} x \mathrm{~d} t \mathrm{~d} y \mathrm{~d} s \\
+\beta|\partial B| \int_{\Omega_{T}} \int_{\Sigma} \alpha(r) u \varphi \mathrm{d} x \mathrm{~d} t \mathrm{~d} r=\left|Y^{*}\right| \int_{\Omega_{T}} f \varphi \mathrm{d} x \mathrm{~d} t+\left|Y^{*}\right| \int_{\Omega} b(x, 0) u_{0}(x) \varphi(x, 0) \mathrm{d} x,
\end{gathered}
$$

for all $\varphi \in \mathcal{C}^{\infty}\left(\overline{\Omega_{T}}\right)$ with supp $\varphi$ bounded away from $\partial \Omega \times(0, T)$ and $t=T$. Since in (4.31) $u$ and $\varphi$, as well as $A^{0}$, do not depend on the microscopic time variables, we readily obtain from it the macroscopic part of (4.30).

Similarly, for $\varphi$ as above and $\psi \in W_{p e r}^{1,2}\left(Y^{*}\right)$, we find reasoning as in Theorem 4.2

$$
\int_{\Omega_{T}} \int_{Q^{*}} A^{0}\left(\nabla u+\nabla_{y} \widetilde{u}\right) \cdot\left(\nabla_{y} \psi\right) \varphi \mathrm{d} x \mathrm{~d} t \mathrm{~d} y \mathrm{~d} s=0
$$

and on integrating over $\Sigma$ this amounts essentially to the microscopic part of (4.30). On invoking the density of the tensor product $\mathcal{C}^{\infty}\left(\overline{\Omega_{T}}\right) \otimes W_{\text {per }}^{1,2}\left(Y^{*}\right)$ in $L^{2}\left(\Omega_{T}, W_{\text {per }}^{1,2}\left(Y^{*}\right)\right)$ we see that $(4.32)$ holds for every $\Psi \in L^{2}\left(\Omega_{T}, W_{p e r}^{1,2}\left(Y^{*}\right)\right)$.

Uniqueness of solution can be proven as in Theorem 4.2. Notice that this does not imply the uniqueness of the function $\widetilde{u}$. Indeed, up to subsequences, in the limit we might obtain different functions satisfying (4.29) and having null $Y$-average; all these functions have also the same $\Sigma$-average, which is part of the unique solution of (4.30).

Remark 4.4. Notice that uniqueness of the solution implies that (4.28) holds true for the whole sequence $u_{\tau}$.

Remark 4.5. We note that the result in Theorem 4.3 holds true also in the case of fast oscillations defined by (4.2) that is (2.40). However in that case we can take 
advantage of estimate (3.17) and of Theorem 2.24, obtaining the stronger result stated in Theorem 4.2 .

Remark 4.6. We remark that we can obtain the limit relations (4.31) and (4.32) even if $A^{\tau}(x, t)=\mathcal{A}^{\tau}\left(x, t, \frac{x}{\varepsilon}, \frac{t}{\tau}\right)$. However in this case we cannot appeal to (2.43) or (2.44) in Theorem 2.24. Then $\widetilde{u}$ may depend on $s$ and in principle one would like to choose also the testing function $\psi$ depending on $s$ in the proof of Theorem 4.3. But this is impossible since the micro-time derivative would yield a term not treatable in the limit. This is why we introduce the averaged $\widetilde{u}_{\Sigma}$ in (4.30), which is possible under assumption (4.27).

As for the case of fast oscillations we give a more precise formulation of (4.30). In this case we factorize

$$
\widetilde{u}_{\Sigma}(x, t, y)=-\nabla_{x} u(x, t) \cdot \sum_{i=1}^{N} \chi_{i}(x, t, y) e_{i}, \quad(x, t, y) \in \Omega_{T} \times Y^{*},
$$

where $\chi_{i}$ for $1 \leq i \leq N$ satisfy $\mathcal{M}_{Y^{*}}\left(\chi_{i}\right)=0$. By using (4.33) in (4.30) and choosing alternatively $\varphi=0$ and $\Psi=0$, we find that $\chi_{i}$ and $u$ satisfy again (4.20)-(4.25). The only difference with the case of fast oscillations is that in this one $A^{0}=A^{0}(x, t, y)$ so that $\mathcal{M}_{\Sigma}\left(A^{0}\right)=A^{0}$ and $\mathcal{M}_{\Sigma}\left(b_{i, j}\right)=b_{i, j}$.

\section{The CASE OF $\sigma / \varepsilon \rightarrow \infty$.}

In this section we assume

$$
\lim _{\tau \rightarrow 0} \frac{\sigma}{\varepsilon}=\infty .
$$

Then the limiting behavior of the sequence $\left\{u_{\tau}\right\}$ is trivial in the following sense.

Theorem 5.1. Let (5.1) hold true. Then the sequence $\left\{u_{\tau}\right\}$ of solutions to (3.6)(3.9) satisfies as $\tau \rightarrow 0$

$$
\mathcal{T}_{\tau}^{*}\left(u_{\tau}\right) \rightarrow 0, \quad \text { strongly in } L^{2}\left(\Omega_{T} \times Q^{*}\right) .
$$

Proof. Since we may appeal to Proposition 3.7 we only need show that $u=0$.

Let us choose an $\alpha_{0}>0$ and an interval $K \subset(0,1)$ such that

$$
\alpha(r) \geq \alpha_{0}>0, \quad r \in K .
$$

Then we go back to (3.15). Unfolding the boundary integral there we get

$$
\begin{aligned}
\gamma & \geq \frac{\sigma}{\varepsilon} \int_{\Omega_{T}} \int_{\partial B} \int_{\Sigma} \alpha(r) \mathcal{T}_{\tau, \sigma}^{b}\left(u_{\tau}\right)^{2}(x, t, y, r) \mathrm{d} x \mathrm{~d} t \mathrm{~d} \sigma_{y} \mathrm{~d} r \\
& \geq \alpha_{0} \frac{\sigma}{\varepsilon} \int_{\Omega_{T}} \int_{\partial B} \int_{K} \mathcal{T}_{\tau, \sigma}^{b}\left(u_{\tau}\right)^{2}(x, t, y, r) \mathrm{d} x \mathrm{~d} t \mathrm{~d} \sigma_{y} \mathrm{~d} r .
\end{aligned}
$$


Then owing to Proposition 2.20, taking into account that (5.1) implies (2.35) with $p=2$, on using (5.4) we get

$$
\begin{array}{rl}
\int_{\Omega_{T}} \int_{\partial B} \int_{K} u(x, t)^{2} & \mathrm{~d} x \mathrm{~d} t \mathrm{~d} \sigma_{y} \mathrm{~d} r \\
\leq \lim _{\tau \rightarrow 0} \int_{\Omega_{T}} \int_{\partial B} \int_{K} \mathcal{T}_{\tau, \sigma}^{b}\left(u_{\tau}\right)^{2}(x, t, y, r) \mathrm{d} x \mathrm{~d} t \mathrm{~d} \sigma_{y} \mathrm{~d} r \leq \lim _{\tau \rightarrow 0} \gamma \frac{\varepsilon}{\sigma}=0 .
\end{array}
$$

\section{REFERENCES}

[1] A. Abenavoli, M.L. Di Francesco, I. Schroeder, S. Epimoshko, S. Gazzarini, U.P. Hansen, G. Thiel, and A. Moroni. Fast and slow gating are inherent properties of the pore module of the $\mathrm{K}^{+}$channel Kcv Journal of General Physiology, 134(3):219-229, 2009.

[2] G. Allaire. Homogenization and two-scale convergence. SIAM Journal on Mathematical Analysis, 23(6):1482-1518, 1992.

[3] M. Amar, D. Andreucci and D. Bellaveglia. The Time-Periodic Unfolding Operator and Applications to Parabolic Homogenization. Preprint, 2015.

[4] M. Amar, A. Dall'Aglio and F. Paronetto. Homogenization of forward-backward parabolic equations. Asymptotic Analysis, 42(1-2):123-132, 2005.

[5] M. Amar, A. Dall'Aglio and F. Paronetto. Homogenization of changing type evolution equations. Journal of Convex Analysis, 12(1):221-237, 2005.

[6] D. Andreucci and D. Bellaveglia. Permeability of interfaces with alternating pores in parabolic problems. Asymptotic Analysis, 79:189-227, 2012.

[7] D. Andreucci, D. Bellaveglia and E.N.M. Cirillo, A model for enhanced and selective transport through biological membranes with alternating pores. Mathematical biosciences, $257: 42-49,2014$.

[8] D. Andreucci, D. Bellaveglia, E.N.M. Cirillo and S. Marconi, Flux through a time-periodic gate: Monte carlo test of a homogenization result. In Proceedings of the 3rd International Conference on Simulation and Modeling Methodologies, Technologies and Applications, pages 626-635, SciTePress, 2013.

[9] A. Becskei and I.W. Mattaj. Quantitative models of nuclear transport. Current Opinion in Cell Biology, 17:27-34, 2005.

[10] G.A. Chechkin, A.L. Piatniski and A.S. Shamev. Homogenization: methods and applications. American Mathematical Society, 234, 2007.

[11] D. Cioranescu, A. Damlamian and G. Griso. The periodic unfolding method in homogenization. SIAM Journal on Mathematical Analysis, 40(4):1585-1620, 2008.

[12] D. Cioranescu, A. Damlamian, P. Donato, G. Griso and R. Zaki. The periodic unfolding method in domains with holes. SIAM Journal on Mathematical Analysis, 44(2):718-760, 2012.

[13] D. Cioranescu, A. Damlamian and G. Griso. Periodic unfolding and homogenization. Comptes Rendus Mathematique, 335(1):99-104, 2002.

[14] D. Cioranescu, A. Damlamian, G. Griso, and D. Onofrei. The periodic unfolding method for perforated domains and Neumann sieve models. Journal de mathématiques pures et appliquées, 89(3):248-277, 2008.

[15] D. Cioranescu and P. Donato. Exact internal controllability in perforated domains. Journal de mathématiques pures et appliquées, 68(2):185-213, 1989. 
[16] D. Cioranescu, P. Donato and R. Zaki. The periodic unfolding method in perforated domains Portugaliae Mathematica, 63(4):467-496, 2006.

[17] D. Cioranescu and F. Murat. Un terme étrange venu dâĂŹailleurs, Non-Linear Partial Differential Equations and Their Applications, Collége de France Seminar, Vols II and III. Research Notes in Mathematics, 60,70:98-138, 154-178, 1982.

[18] D. Cioranescu and J.S.J. Paulin. Homogenization in open sets with holes. Journal of mathematical analysis and applications, 71(2):590-607, 1979.

[19] D. Cioranescu and A.L. Piatnitski. Homogenization in perforated domains with rapidly pulsating perforations. Control, Optimisation and Calculus of Variations, 9:461-483, 2003.

[20] C. Conca and P. Donato. Non-homogeneous Neumann problems in domains with small holes. RAIRO-Modélisation mathématique et analyse numérique, 22(4):561-607, 1988.

[21] O.A. Ladyzenskaja, V.A. Solonnikov, and N.N. Ural'ceva. Linear and quasi-linear equations of parabolic type. volume 23. American Mathematical Society, 1968.

[22] J.L. Lions. Asymptotic expansions in perforated media with a periodic structure. Rocky Mountain J. Math, 10(1):125-140, 1980.

[23] L. Tartar. The general theory of homogenization. A personalized introduction, volume 7 of Lecture Notes of the Unione Matematica Italiana. Springer-Verlag, Berlin, 2009.

[24] A.M.J. VanDongen. K channel gating by an affinity-switching selectivity filter. Proceedings of the National Academy of Sciences of the United States of America, 101(9):3248-3252, 2004. 